Quant um mechani cal and sem cl assi cal st udy of the coll i near three-body Coul ont probl em I nel asti c colli si ons bel ow the three-body di si nt egr at $i$ on threshol $d$

\begin{tabular}{|c|c|}
\hline $\begin{array}{l}\text { jour nal or } \\
\text { publ i cat } i \text { on title }\end{array}$ & PHYSI CAL REVI EW A \\
\hline vol une & Vol . 70 \\
\hline number & I ssue 6 \\
\hline page $r$ ange & $062721-1-062721-12$ \\
\hline year & $200412-01$ \\
\hline URL & ht t p: //hdl . handl e. net $/ 10655 / 320$ \\
\hline
\end{tabular}




\title{
Quantum-mechanical and semiclassical study of the collinear three-body Coulomb problem: Inelastic collisions below the three-body disintegration threshold
}

\author{
Oleg I. Tolstikhin \\ Russian Research Center “Kurchatov Institute,” Kurchatov Square 1, Moscow 123182, Russia \\ Chusei Namba \\ National Institute for Fusion Science, Toki 509-5292, Japan \\ (Received 26 June 2004; published 28 December 2004)
}

\begin{abstract}
A quantum-mechanical (QM) and semiclassical (SC) study of inelastic collisions in collinear three-body Coulomb systems below the three-body disintegration threshold is presented. The QM results are obtained by solving the stationary Schrödinger equation in hyperspherical coordinates using the slow/smooth variable discretization method. After appropriate rescaling of the hyperspherical coordinates, an asymptotic parameter $0 \leqslant h \leqslant 1$ that depends only on the masses of particles and has the meaning of an effective Planck's constant for the motion in hyperradius emerges. The SC results are obtained in the leading order approximation of the asymptotic expansion in $h$. The main attention is paid to investigating how the SC and QM results converge as $h \rightarrow 0$. It is shown that the overall agreement for a wide spectrum of systems and processes is surprisingly good even for $h \sim 1$. However, because of interference effects the convergence is not monotonic, and the SC results may be grossly in error in the situations where a destructive interference occurs. The analysis of hidden crossings clarifies mechanisms of the nonadiabatic transitions. It is shown that if the oppositely charged particle is located between the two others, the nonadiabatic transitions occur near the top of the potential barrier via the well-known $T$ series of hidden crossings. If it is located on one end of the system, then there is no potential barrier for real values of the angular variable, but there still exists an extremum in the complex plane; the mechanism of nonadiabatic transitions in this case is called the complex $T$ series of hidden crossings.
\end{abstract}

DOI: $10.1103 /$ PhysRevA.70.062721

PACS number(s): 34.10.+x, 31.15.Ja, 31.15.Gy

\section{INTRODUCTION}

Systems in which one degree of freedom can be treated as "classical" (or "slow") while the others remain "quantal" (or "fast") are of great interest for further development of quantum theory and its applications. The selected degree of freedom is characterized by higher rate of the accumulation of action, which can be explained by the presence of a small parameter $h$ having the meaning of an effective Planck's constant for the motion in the corresponding variable. Therefore an adequate theoretical approach to such systems is the asymptotic solution of the problem for $h \longrightarrow 0$, i.e., the semiclassical approximation. In this approach, "quantal" degrees of freedom are eliminated by the expansion in the adiabatic basis and the resulting set of ordinary differential equations describing the motion in the "classical" variable is solved by asymptotic methods. Historically, the first application of such an approach was the treatment of the rotational and vibrational spectra of diatomic molecules by Born and Oppen heimer [1]; the selected variable here is the internuclear distance and the small parameter is provided by the electron-tonucleus mass ratio. The term semiclassical (or quasiclassical, in Russian tradition) is commonly used for this kind of approximation in time-independent formulations [2-5]. A similar theoretical scheme in time-dependent problems, in which case the selected variable is time and the small parameter is the characteristic velocity of "slow" subsystem or the rate of variation of external conditions, is usually called the adiabatic approach [6]. It has been pioneered by Born and Fock [7] who derived the first definite, albeit negative, result concerning the probability of nonadiabatic transitions (the adiabatic theorem). We consider the terms semiclassical and adiabatic in the above context essentially as synonyms whose exact meaning is asymptotic. Because our analysis is based on the stationary Schrödinger equation, we adopt the time-independent terminology.

The main virtue of the semiclassical (SC) theory is that it not only enables one to obtain an approximate quantitative description of the dynamics in the situations where an accurate quantum-mechanical (QM) solution is not accessible, but also clarifies its physical mechanisms. However, SC results have limited accuracy and usually cannot be improved. From the pragmatic viewpoint, accuracy is eventually the key factor that determines whether such results are meaningful and useful or not. An asymptotic estimate of the error is usually available, e.g., $O(h)$, which, however, tells nothing about its actual numerical magnitude. In order to appreciate how small $O(h)$ is for the given class of problems it is desirable to study some simplified but still realistic model for which a reliable QM solution can be obtained and simultaneously an SC solution is expected to be valid and to analyze how these two solutions converge with each other as the asymptotic parameter $h$ tends to zero. A study of this type is presented in this paper.

The class of problems we have in mind, towards which our analysis is directed, is low energy collisions in threebody Coulomb systems. The model we chose to consider is the collinear three-body Coulomb problem. The stationary Schrödinger equation describing this model allows a very accurate numerical solution in hyperspherical coordinates. At 
the same time, after appropriate rescaling of the hyperspherical coordinates, the Schrödinger equation can be presented in such a form that an effective Planck's constant $h$ for the motion in hyperradius emerges. The parameter $h$ depends only on the masses of particles, is dimensionless, and varies in the interval $0 \leqslant h \leqslant 1$. The SC solution becomes exact for $h \rightarrow 0$, so by varying $h$ one can observe how the SC and QM results converge. To analyze this convergence is one of the goals of this study. We believe that the conclusions made in this respect should remain valid also for three-dimensional systems for which, however, such an analysis would be much more laborious. The other goal is to discuss mechanisms of the collinear dynamics. These results cannot be transferred directly to the three-dimensional case, but present a necessary first step in that direction.

There exists a great number of studies devoted to the comparison of SC and QM results for various physical systems, especially in the field of slow atomic and molecular collisions, see [2-5] and references therein. In this situation, it is natural to ask what is the reason for undertaking yet another study of this type. We answer this question by emphasizing some features of the collinear three-body Coulomb problem that distinguish it from the majority of other models considered earlier. First, it reproduces all the spectrum of states and processes possible in three-body Coulomb systems in three dimensions-this is what was meant above by a realistic model. Second, it has two degrees of freedom (this is the minimum number of degrees of freedom for a model to be realistic) which, in contrast to various two-state and multistate models [8-10], will be treated explicitly. Finally, it contains a continuously changeable small parameter $h$. The existence of an explicit small parameter is not that important for obtaining an SC solution for one particular system, but it is essential for understanding how the $\mathrm{SC}$ and $\mathrm{QM}$ results converge.

The paper is organized as follows. Basic equations describing the collinear three-body Coulomb problem are formulated in Sec. II. The problem contains four parameters, two mass ratios and two charge ratios; in addition, two physically different arrangements of three particles on a line are possible, depending on where the oppositely charged particle is located. In this paper, we restrict our consideration to two particular families of systems, one for each of the two arrangements, whose members differ only by the value of $h$. Our QM treatment is based on the slow/smooth variable discretization (SVD) method [11]. The SVD suggests a very efficient approach to the numerical solution of the Schrödinger equation for systems with one "classical" degree of freedom, as is confirmed by the growing number of its applications in hyperspherical studies of two- [12] and three[13] electron atoms, ion-atom collisions [14], three-body Coulomb systems involving exotic particles [15,16], and chemical reactions $[17,18]$. Because a detailed description of the program used to obtain the QM results has been published recently [19], we only briefly outline its structure in Sec. III. On the contrary, our SC treatment is discussed rather in detail in Sec. IV. There are two basic questions that should be answered by the theory: how to calculate the probabilities of transitions and what are their mechanisms. An answer to the first question was given by Landau [20], who showed
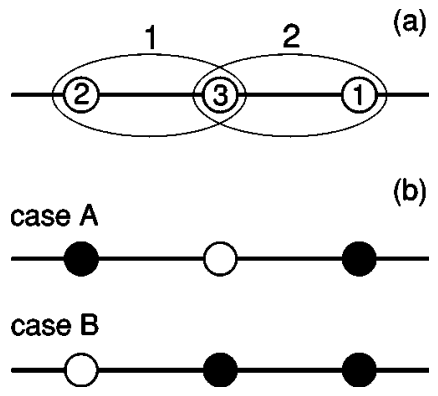

FIG. 1. Three particles on a line. (a) The convention for their numbers; this order does not change during the motion. (b) Two physically different variants of their relative position; open and closed circles symbolize oppositely charged particles.

that in the SC approximation probabilities of nonadiabatic transitions are determined by crossing points of the adiabatic potentials and are typically exponentially small, and Stueckelberg [21], who in the same year published a solution to the generic two-state problem. An answer to the second question should be sought in the properties of the adiabatic basis, namely, in the phenomenon of hidden crossings discovered by Solov'ev [22], see also [6,23] and references therein. Accordingly, our SC treatment consists of two parts: a description of the procedure used to obtain the SC results (Secs. IV A-IV D) and the analysis of hidden crossings (Sec. IV E). Section V presents the results of scattering calculations. Section VI concludes the paper.

\section{THE COLLINEAR THREE-BODY COULOMB PROBLEM}

We consider a system of three particles with masses $m_{i}$ and charges $e_{i}, i=1,2,3$, constrained to move along a straight line and interacting via the Coulomb forces. Particles will be called by their numbers, and pairs of particles, by the number of the remaining particle. It should be assumed that particles cannot penetrate through each other in collisions (see the discussion of boundary conditions below), so they preserve their order on the line. We enumerate them as shown in Fig. 1(a). Only the case when one of the particles is charged oppositely to the others will be considered. By convention we assume that $e_{2} e_{3}<0$, i.e., the interaction in pair 1 is attractive. Then there are two possibilities, either $e_{1} e_{3}<0$ or $e_{1} e_{3}>0$, which will be referred to as cases $\mathrm{A}$ and $\mathrm{B}$, respectively, see Fig. 1(b). In case A, the system can disintegrate into a free particle and a bound pair in two ways, $(23)+1$ and $2+(31)$, which will be called arrangements 1 and 2 , while in case B such disintegration can occur only in arrangement 1 . Case $\mathrm{A}$ under the condition that particles 1 and 2 are identical, i.e., $m_{1}=m_{2}$ and $e_{1}=e_{2}$, will be called the symmetric case.

The stationary Schrödinger equation for this system reads

$$
(T+V-E) \Psi=0,
$$

where $T$ is the kinetic energy, 


$$
T=-\frac{\hbar^{2}}{2}\left(\frac{\partial^{2}}{\partial x_{\alpha}^{2}}+\frac{\partial^{2}}{\partial y_{\alpha}^{2}}\right)=-\frac{\hbar^{2}}{2}\left(\frac{1}{R} \frac{\partial}{\partial R} R \frac{\partial}{\partial R}+\frac{1}{R^{2}} \frac{\partial^{2}}{\partial \phi^{2}}\right),
$$

$V$ is the Coulomb potential energy,

$$
\begin{aligned}
V= & \frac{e_{3} e_{2}}{X_{3}-X_{2}}+\frac{e_{1} e_{3}}{X_{1}-X_{3}}+\frac{e_{1} e_{2}}{X_{1}-X_{2}}=\frac{z_{1}}{y_{1}}+\frac{z_{2}}{x_{1} \sin \gamma_{3}-y_{1} \cos \gamma_{3}} \\
& +\frac{z_{3}}{x_{1} \sin \gamma_{2}+y_{1} \cos \gamma_{2}}=\frac{z_{1}}{x_{2} \sin \gamma_{3}-y_{2} \cos \gamma_{3}}+\frac{z_{2}}{y_{2}} \\
& +\frac{z_{3}}{x_{2} \sin \gamma_{1}+y_{2} \cos \gamma_{1}}=\frac{C(\phi)}{R},
\end{aligned}
$$

$C(\phi)$ is the effective charge,

$$
C(\phi)=\frac{z_{1}}{\sin \phi}+\frac{z_{2}}{\sin \left(\gamma_{3}-\phi\right)}+\frac{z_{3}}{\sin \left(\gamma_{2}+\phi\right)},
$$

and $E$ is the total energy measured from the three-body disintegration threshold. The different sets of coordinates used here are defined as follows. $X_{i}$ are the coordinates of particles on the line with the origin at the center of mass, thus $m_{1} X_{1}$ $+m_{2} X_{2}+m_{3} X_{3}=0$. $x_{\alpha}$ and $y_{\alpha}, \alpha=1,2$, are two sets of the mass-scaled Jacobi coordinates,

$$
\begin{gathered}
x_{1}=\sqrt{\frac{m_{1} m_{\mathrm{tot}}}{m_{2}+m_{3}}} X_{1}, \\
y_{1}=\sqrt{\frac{m_{2} m_{3}}{m_{2}+m_{3}}}\left(X_{3}-X_{2}\right),
\end{gathered}
$$

and

$$
\begin{gathered}
x_{2}=-\sqrt{\frac{m_{2} m_{\mathrm{tot}}}{m_{1}+m_{3}}} X_{2}, \\
y_{2}=\sqrt{\frac{m_{1} m_{3}}{m_{1}+m_{3}}}\left(X_{1}-X_{3}\right),
\end{gathered}
$$

related to each other by the kinematic rotation [24]

$$
\left(\begin{array}{l}
x_{2} \\
y_{2}
\end{array}\right)=\left(\begin{array}{cc}
\cos \gamma_{3} & \sin \gamma_{3} \\
\sin \gamma_{3} & -\cos \gamma_{3}
\end{array}\right)\left(\begin{array}{l}
x_{1} \\
y_{1}
\end{array}\right) \text {. }
$$

Finally, $R$ and $\phi$ are the hyperspherical coordinates,

$$
\begin{gathered}
R=\sqrt{x_{1}^{2}+y_{1}^{2}}=\sqrt{x_{2}^{2}+y_{2}^{2}}, \\
\phi=\arctan \left(y_{1} / x_{1}\right)=\gamma_{3}-\arctan \left(y_{2} / x_{2}\right) .
\end{gathered}
$$

In these equations, $z_{i}$ are the pair charges,

$$
z_{i}=e_{i+1} e_{i+2} \sqrt{\frac{m_{i+1} m_{i+2}}{m_{i+1}+m_{i+2}}},
$$

$\gamma_{i}$ are the angles of kinematic rotations,

$$
\gamma_{i}=\arctan \sqrt{\frac{m_{i} m_{\mathrm{tot}}}{m_{i+1} m_{i+2}}}, \quad 0 \leqslant \gamma_{i} \leqslant \pi / 2,
$$

where $(i, i+1, i+2)$ is a cyclic permutation of $(1,2,3)$, and $m_{\mathrm{tot}}=m_{1}+m_{2}+m_{3}$. The volume element in configuration space is $d \mathcal{V}=d x_{\alpha} d y_{\alpha}=R d R d \phi$.
Let us discuss the boundary conditions. Equation (1) should be considered in the region $-\infty<X_{2} \leqslant X_{3} \leqslant X_{1}<\infty$ that in hyperspherical coordinates is represented by the sector

$$
0 \leqslant R<\infty, \quad 0 \leqslant \phi \leqslant \gamma_{3} .
$$

Its boundaries correspond to binary collisions in pairs $1(\phi$ $=0)$ and $2\left(\phi=\gamma_{3}\right)$, and its apex $(R=0)$ represents the point of triple collision. In order that matrix elements of the potential energy (3) be finite, the solutions of Eq. (1) must vanish at the points of interparticle collisions. Therefore one should require

$$
\left.\Psi(R, \phi)\right|_{\phi=0}=\left.\Psi(R, \phi)\right|_{\phi=\gamma_{3}}=0 .
$$

This boundary condition justifies our assumption that particles cannot penetrate through each other; it is specific to one-dimensional Coulomb problems, the situation in spaces of higher dimension is different. In the symmetric case, the solutions of Eq. (1) are either even $(\sigma=+)$ or odd $(\sigma=-)$ under the permutation of identical particles 1 and 2 , so it is sufficient to consider only a half of the sector (10) with the additional boundary condition

$$
\begin{gathered}
\left.\frac{\partial \Psi(R, \phi)}{\partial \phi}\right|_{\phi=\gamma_{3} / 2}=0 \text { for } \sigma=+, \\
\left.\Psi(R, \phi)\right|_{\phi=\gamma_{3} / 2}=0 \text { for } \sigma=-.
\end{gathered}
$$

The boundary conditions in the asymptotic region $R \rightarrow \infty$ depend on the energy $E$. In this paper we consider only inelastic collisions below the three-body disintegration threshold. In case $\mathrm{A}$, there are three types of such processes: excitation in arrangement $\alpha$,

$$
\begin{array}{ll}
(23)_{\nu}+1 \leftrightarrow(23)_{\mu}+1, & \alpha=1, \\
2+(31)_{\nu} \leftrightarrow 2+(31)_{\mu}, & \alpha=2,
\end{array}
$$

and rearrangement,

$$
(23)_{\nu}+1 \leftrightarrow 2+(31)_{\mu},
$$

where indices $\nu$ and $\mu$ identify states of the bound pair in the initial and final states of the system. Threshold energies for various scattering channels are given by

$$
E_{\nu}^{(\alpha)}=\frac{z_{\alpha}^{2} E_{\nu}}{\hbar^{2}}, \quad \alpha=1,2, \quad \nu=1,2, \ldots,
$$

where $E_{\nu}$ denotes the hydrogenic spectrum,

$$
E_{\nu}=-\frac{1}{2 \nu^{2}} \text {. }
$$

By convention we assume that $\left|z_{1}\right| \geqslant\left|z_{2}\right|$, hence $E_{\nu}^{(1)} \leqslant E_{\nu}^{(2)}$, which is always possible to achieve by interchanging similarly charged particles 1 and 2 . The asymptotic boundary conditions for processes (13) in the energy range $E<0$ can be formulated in a standard way; we do not reproduce them here, for details see Ref. [19]. The scattering matrix $\mathbf{S}$ can be divided into four blocks $\mathbf{S}^{(\alpha \beta)}$ with elements $S_{\nu \mu}^{(\alpha \beta)}$, where the 
upper indices refer to arrangements and the lower ones to states. In the symmetric case $\mathbf{S}^{(11)}=\mathbf{S}^{(22)}$ and $\mathbf{S}^{(12)}=\mathbf{S}^{(21)}$, and it is more convenient to consider a symmetrized scattering matrix $\mathbf{S}^{(\sigma)}$ defined by

$$
\mathbf{S}^{( \pm)}=\mathbf{S}^{(11)} \pm \mathbf{S}^{(12)} .
$$

In case $\mathrm{B}$, only processes (13a) in arrangement $\alpha=1$ are possible, so $\mathbf{S}=\mathbf{S}^{(11)}$.

\section{QUANTUM-MECHANICAL TREATMENT}

The QM description of processes (13) can be obtained by solving the Schrödinger equation (1) numerically. These days such a solution is quite feasible, although for obtaining reliable results the experience of the researcher still matters more than the power of the computer. Recently we have developed a program СтвC that enables one to solve the collinear three-body Coulomb problem for a wide spectrum of systems and processes in the energy range below the threebody disintegration threshold [19]. Because major computational technologies used in СТВC have already been described in the literature, here we only briefly outline its general structure. In the internal region $R \leqslant R_{\mathrm{m}}$, Eq. (1) is solved in terms of hyperspherical coordinates using the SVD method [11] in combination with the $R$-matrix propagation technique [25]. In the external region $R \geqslant R_{\mathrm{m}}$, Eq. (1) is solved separately in each attractive Coulomb valley extending along the directions $y_{\alpha}=0$ in terms of the appropriate set of Jacobi coordinates using the asymptotic expansions developed in [26,27] and implemented in [28]. Internal and external solutions are then matched along the arc $R=R_{\mathrm{m}}$ to construct a global solution from which the scattering matrix is obtained. More details on the program and results of illustrative calculations for a number of systems and processes can be found in [19]. We wish to emphasize that in spite of swift brevity of this section the numerical solution of Eq. (1) by no means should be considered as something of secondary importance in this work. On the contrary, it is availability of accurate QM results that makes the present attempt to appreciate the quality of the SC solution possible.

\section{SEMICLASSICAL TREATMENT}

\section{A. Scaling}

Let us introduce scaled hyperspherical coordinates,

$$
\widetilde{R} \equiv h \hbar^{-2}\left|z_{1}\right| R, \quad \widetilde{\phi} \equiv h^{-1} \phi,
$$

scaled energies,

$$
\left(\widetilde{E}, \widetilde{E}_{\nu}^{(\alpha)}\right) \equiv \hbar^{2}\left|z_{1}\right|^{-2}\left(E, E_{\nu}^{(\alpha)}\right)
$$

and scaled charges,

$$
\widetilde{C} \equiv h\left|z_{1}\right|^{-1} C, \quad \widetilde{z_{i}} \equiv\left|z_{1}\right|^{-1} z_{i},
$$

where

$$
h=\frac{2 \gamma_{3}}{\pi}, \quad 0 \leqslant h \leqslant 1 .
$$

After scaling, all the quantities under consideration become dimensionless with the units of hyperradius and energy given by

$$
R_{0}=\frac{\hbar^{2}}{h\left|z_{1}\right|}, \quad E_{0}=\frac{z_{1}^{2}}{\hbar^{2}} .
$$

These units characterize pair $1: R_{0}$ estimates the value of hyperradius on the boundary between the regions of condensation (all particles are so close together that the kinetic energy dominates over the potential one) and fragmentation in arrangement 1 (pair 1 in the ground state and particle 1 are separated), and $E_{0}$ defines the energies of bound states in pair 1. Similar units could be defined in case A for pair 2, but in case B there are no bound states in pair 2, so such units would lose their meaning. Thus $R_{0}$ and $E_{0}$ are natural units for the problem. Only scaled quantities will be considered in the following, so from here on we omit the tilde. The effective charge is now explicitly given by

$$
C(\phi)=-\frac{h}{\sin [h \phi]}+\frac{h z_{2}}{\sin [h(\pi / 2-\phi)]}+\frac{h z_{3}}{\sin \left[\gamma_{2}+h \phi\right]},
$$

the threshold energies are

$$
E_{\nu}^{(1)}=E_{\nu}, \quad E_{\nu}^{(2)}=z_{2}^{2} E_{\nu},
$$

and the pair charges are (note that after scaling $z_{1}=-1$ )

$$
z_{2}=-\frac{e_{1}}{e_{2}} \sqrt{\frac{m_{1}\left(m_{2}+m_{3}\right)}{m_{2}\left(m_{1}+m_{3}\right)}}, \quad z_{3}=-\frac{e_{1}}{e_{3}} \sqrt{\frac{m_{1}\left(m_{2}+m_{3}\right)}{m_{3}\left(m_{1}+m_{2}\right)}} .
$$

According to our conventions, $-1 \leqslant z_{2}<0$ in case $\mathrm{A}$ and $z_{2}$ $>0$ in case $\mathrm{B} ; z_{3}$ may have arbitrary value, but its sign is opposite to that of $z_{2}$.

One reason for scaling (17a)-(17c) is to bring systems with vastly different masses and/or charges of particles as far as possible to a common scale in configuration and energy spaces. But there is also a deeper goal: scaling reveals the asymptotic parameter $h$ essential for the SC treatment of the problem. A resemblance of this notation to Planck's constant is not accidental. Indeed, in scaled hyperspherical coordinates the Schrödinger equation (1) reads

$$
\left[-\frac{h^{2}}{2 R} \frac{\partial}{\partial R} R \frac{\partial}{\partial R}-\frac{1}{2 R^{2}} \frac{\partial^{2}}{\partial \phi^{2}}+\frac{C(\phi)}{R}-E\right] \Psi(R, \phi)=0,
$$

thus $h$ has the meaning of an effective Planck's constant for the motion in $R$. The configuration space (10) is now defined by

$$
0 \leqslant R<\infty, \quad 0 \leqslant \phi \leqslant \pi / 2,
$$

so its boundaries do not depend anymore on $h$. The effective charge (20) does depend on $h$, explicitly and via its dependence on the masses of particles that can be converted into a 
dependence on $h$ and the ratio $m_{1} / m_{2}$. However, this dependence does not modify the asymptotic structure of the problem because for any fixed value of $m_{1} / m_{2}$

$$
\left.C(\phi)\right|_{h \rightarrow 0}=-\frac{1}{\phi}-\frac{e_{1}}{e_{2}(\pi / 2-\phi)}-\frac{2 e_{1}}{\pi e_{3}}+O\left(h^{2}\right) .
$$

Thus in the limit $h \rightarrow 0$ the parameter $h$ enters Eq. (23) only in the form of an effective Planck's constant for the motion in $R$, which renders this equation amenable to the analysis by asymptotic methods. As follows from Eqs. (9) and (18), $h$ depends only on the masses of particles and $h \ll 1$ corresponds to $m_{3} \ll \min \left(m_{1}, m_{2}\right)$, therefore our SC treatment is expected to yield exact results in the limit of heavy-lightheavy systems.

As can be seen from Eqs. (20) and (22), besides $h$ there are three more parameters in the problem, one mass ratio $m_{1} / m_{2}$ and two charge ratios $e_{1} / e_{2}$ and $e_{1} / e_{3}$. To keep the length of the paper finite, we consider two particular families of systems, one for each of the two cases A and B. Systems in these families differ only by the value of $h$, while the other parameters are fixed by the conditions $e_{1}=e_{2}=-e_{3}$ in case $\mathrm{A}$, $e_{1}=-e_{2}=e_{3}$ in case $\mathrm{B}$, and in both cases $m_{1}=m_{2}=M m_{3}$, where the mass ratio $M$ is related to $h$ by

$$
M=\frac{\cos (\pi h / 2)}{1-\cos (\pi h / 2)}, \quad h=\frac{2}{\pi} \arctan \frac{\sqrt{1+2 M}}{M} .
$$

Three representative combinations of the masses with $M$ $=\infty$ (heavy-light-heavy, e.g., pep in case A and $p e \bar{p}$ in case B), $M=1$ (equal masses, e.g., $e e^{+} e$ in case $\mathrm{A}$ and $e^{+} e e$ in case $\mathrm{B}$ ), and $M=0$ (light-heavy-light, e.g., epe in case A and $e p e^{+}$ in case B) correspond to $h=0,2 / 3$, and 1 , respectively. The effective charge (20) for these families takes the form

$$
\begin{aligned}
C(\phi)= & -\frac{h}{\sin [h \phi]} \mp \frac{h}{\sin [h(\pi / 2-\phi)]} \\
& \pm \frac{h}{2 \sin (\pi h / 4) \cos [h(\phi-\pi / 4)]} \\
= & -\frac{1}{\phi} \mp \frac{1}{\pi / 2-\phi} \pm \frac{2}{\pi}+O\left(h^{2}\right),
\end{aligned}
$$

where the upper (lower) signs stand for case A (B). It has an attractive singularity at $\phi=0$ and another attractive (repulsive) singularity in case A (B) at $\phi=\pi / 2$ that originate from the Coulomb interactions in pairs 1 and 2, respectively, see Fig. 2. Function (27a) only weakly depends on $h$ being very close to its limiting form (27b) for all values of $h$ in the interval (18). For these systems $\left|z_{2}\right|=1$, so the threshold energies (21) are simply given by the hydrogenic spectrum,

$$
E_{\nu}^{(1)}=E_{\nu}^{(2)}=E_{\nu} .
$$

Note that the systems in case A are symmetric; we shall consider two permutation symmetries $\sigma= \pm$ separately.

\section{B. Primitive semiclassical solutions}

Keeping in mind possible applications of the present approach to the three-body Coulomb problem in three dimen-
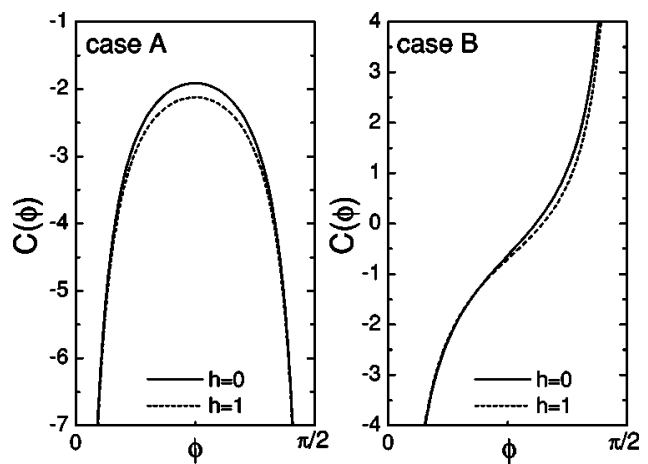

FIG. 2. The effective charge (27a) for two extreme values of $h$ in cases A and B. The solid curves correspond to Eq. (27b).

sions, without complicating the analysis in this section instead of Eq. (23) we consider a more general equation,

$$
\left[-\frac{h^{2}}{2 R^{d-1}} \frac{\partial}{\partial R} R^{d-1} \frac{\partial}{\partial R}+\frac{\Lambda^{2}}{2 R^{2}}+\frac{C(\Omega)}{R}-E\right] \Psi(R, \Omega)=0 .
$$

This equation describes a few-body Coulomb system with $d$-dimensional configuration space parameterized by hyperspherical coordinates $(R, \Omega)$, where $\Omega$ denotes a set of $d-1$ angular variables and $\Lambda$ is the grand angular momentum operator [29]. A relation with the notation above is established by $d=2, \Omega=\phi$, and $\Lambda^{2}=-\partial^{2} / \partial \phi^{2}$. It will be assumed that the effective charge $C(\Omega)$ does not depend on the asymptotic parameter $h$.

Let us introduce some notation related to the adiabatic basis. The adiabatic Hamiltonian for Eq. (29) is

$$
\mathcal{U}(R)=\frac{1}{2} \Lambda^{2}+R C(\Omega) .
$$

Its eigenfunctions defined by

$$
\left[\mathcal{U}(R)-U_{\nu}(R)\right] \Phi_{\nu}(\Omega ; R)=0, \quad \nu=1,2, \ldots,
$$

and satisfying the regularity boundary conditions on the hypersphere constitute the adiabatic basis. We normalize them by $\left\langle\Phi_{\nu}(\Omega ; R) \mid \Phi_{\mu}(\Omega ; R)\right\rangle=\delta_{\nu \mu}$, where $\langle\cdots\rangle$ denotes integration over $\Omega$. Along with the adiabatic eigenvalues $U_{\nu}(R)$, it is convenient to introduce the adiabatic potentials $V_{\nu}(R)$, effective quantum numbers $N_{\nu}(R)$, and momenta $K_{\nu}(R)$,

$$
\begin{gathered}
V_{\nu}(R)=U_{\nu}(R) / R^{2}, \\
N_{\nu}(R)=\left[-2 V_{\nu}(R)\right]^{-1 / 2}, \\
K_{\nu}(R)=\left\{2\left[E-V_{\nu}(R)\right]\right\}^{1 / 2} .
\end{gathered}
$$

The matrices of nonadiabatic couplings are given by

$$
\begin{gathered}
P_{\nu \mu}(R)=\left\langle\Phi_{\nu}(\Omega ; R)|\partial / \partial R| \Phi_{\mu}(\Omega ; R)\right\rangle, \\
Q_{\nu \mu}(R)=\left\langle\Phi_{\nu}(\Omega ; R)\left|\partial^{2} / \partial R^{2}\right| \Phi_{\mu}(\Omega ; R)\right\rangle,
\end{gathered}
$$

and the nonadiabatic corrections to the adiabatic potentials are 


$$
v_{\nu}(R)=\frac{D}{R^{2}}-\frac{1}{2} Q_{\nu \nu}(R), \quad D=\frac{(d-1)(d-3)}{8} .
$$

Let us introduce a new function,

$$
\psi(R, \Omega)=R^{(d-1) / 2} \Psi(R, \Omega),
$$

and rewrite Eq. (29) as

$$
\left[-\frac{h^{2}}{2} \frac{\partial^{2}}{\partial R^{2}}+\frac{\mathcal{U}(R)+h^{2} D}{R^{2}}-E\right] \psi(R, \Omega)=0 .
$$

The asymptotic solutions to this equation for $h \rightarrow 0$ can be sought in many different forms. Being interested only in the leading order terms, we adopt the following simplest ansatz:

$$
\psi(R, \Omega)=\exp \left[i h^{-1} S_{0}(R)+i S_{1}(R)\right] \varphi(R, \Omega)
$$

where

$$
\varphi=\varphi_{0}+h \varphi_{1}+\cdots
$$

and functions $S_{0}, S_{1}$, and all the $\varphi_{k}$ do not depend on $h$. It is clear that the term $S_{1}$ could be removed from the exponent in Eq. (37a) by absorbing it into the $\varphi$. To eliminate this ambiguity we require $\left\langle\varphi_{0} \mid \varphi_{0}\right\rangle=1$. Substituting Eqs. (37a) and (37b) into Eq. (36) and collecting terms of the same order in $h$, in the zeroth order we have

$$
\left[\frac{1}{2} S_{0}^{\prime 2}+\frac{\mathcal{U}(R)}{R^{2}}-E\right] \varphi_{0}=0,
$$

where the prime denotes differentiation in $R$. Thus $\varphi_{0}$ must be an eigenfunction of $\mathcal{U}(R)$, and then $S_{0}$ is determined by the corresponding eigenvalue. Consider the solution

$$
S_{0}= \pm \int^{R} K_{\nu}(R) d R, \quad \varphi_{0}=\Phi_{\nu}(\Omega ; R)
$$

In the first order we obtain

$$
\left[-i S_{0}^{\prime} \frac{\partial}{\partial R}+S_{0}^{\prime} S_{1}^{\prime}-\frac{i}{2} S_{0}^{\prime \prime}\right] \varphi_{0}+\left[\frac{1}{2} S_{0}^{\prime 2}+\frac{\mathcal{U}(R)}{R^{2}}-E\right] \varphi_{1}=0 .
$$

From this we find

$$
S_{1}=\frac{i}{2} \ln S_{0}^{\prime}
$$

and

$$
\varphi_{1}= \pm i c(R) \Phi_{\nu}(\Omega ; R) \pm i K_{\nu}(R) \sum_{\mu \neq \nu} \frac{P_{\nu \mu}(R)}{V_{\nu}(R)-V_{\mu}(R)} \Phi_{\mu}(\Omega ; R)
$$

The coefficient $c(R)$ here remains undefined. It is determined by the equation arising in the second order of the expansion, which yields

$$
c(R)=\int^{R}\left[\frac{3 K_{\nu}^{\prime 2}}{8 K_{\nu}^{3}}-\frac{K_{\nu}^{\prime \prime}}{4 K_{\nu}^{2}}-\frac{v_{\nu}}{K_{\nu}}-\sum_{\mu \neq \nu} \frac{K_{\nu} P_{\nu \mu}^{2}}{V_{\nu}-V_{\mu}}\right] d R .
$$

This process could be continued to obtain higher terms in Eq. (37b). Summarizing the results, the primitive SC solutions of Eq. (36) are given by

$$
\psi_{\nu}^{ \pm}(R, \Omega)=\frac{\exp \left( \pm i h^{-1} \int_{\nu}^{R} K_{\nu}(R) d R\right)}{K_{\nu}^{1 / 2}(R)} \Phi_{\nu}(\Omega ; R),
$$

while Eqs. (42) and (43) give an estimate of the error term.

It is useful to take a look at these results from a different side. The solutions of Eq. (36) can be expanded in the adiabatic basis as

$$
\psi(R, \Omega)=\sum_{\nu} F_{\nu}(R) \Phi_{\nu}(\Omega ; R)
$$

Substituting this into Eq. (36), one obtains a set of ordinary differential equations for $F_{\nu}(R)$,

$$
\begin{aligned}
& {\left[-\frac{h^{2}}{2} \frac{d^{2}}{d R^{2}}+V_{\nu}(R)-E\right] F_{\nu}(R)} \\
& \quad=-h^{2} v_{\nu}(R) F_{\nu}(R)+h^{2} \sum_{\mu \neq \nu}\left[P_{\nu \mu}(R) \frac{d}{d R}+\frac{1}{2} Q_{\nu \mu}(R)\right] F_{\mu}(R) .
\end{aligned}
$$

In the hyperspherical approach proposed by Macek [30] one seeks accurate numerical solutions to these equations. This approach has proven to be very efficient and is currently widely used for solving various few-body problems in atomic [31] and molecular [32] physics. It is easy to see that the primitive SC solutions (44) can be obtained by neglecting the right-hand sides of Eqs. (46) and solving the resulting decoupled equations using the SC approximation. The purpose of an alternative derivation discussed above was to emphasize an intimate relation between the SC approximation and the adiabatic basis [1]. Indeed, we have seen that the asymptotic expansion (37a) and (37b) singles out the adiabatic basis. On the other hand, any complete angular basis can be used to expand the accurate QM solution; the adiabatic basis in this case merely has an advantage of faster convergence.

Our leading order approximation is based on the primitive SC solutions (44). As can be seen from Eqs. (46), the nonadiabatic correction (34) adds to the adiabatic potential $V_{\nu}(R)$ a term $O\left(h^{2}\right)$. There is another contribution of the same order that has not been taken into account above-the so-called Langer correction [33] arising from the fact that $V_{\nu}(R)$ diverges $\propto 1 / R^{2}$ as $R \rightarrow 0$. In the leading order approximation both corrections must be neglected because their effect on the wave function is of the same order as Eq. (42). This means that in the leading order approximation the adiabatic potentials $V_{\nu}(R)$ are effectively defined with an intrinsic error $O\left(h^{2}\right)$, which leads to a similar error $O\left(h^{2}\right)$ in the energies of bound and resonance states and to an error $O(h)$ in the elastic 
phase shifts. However, in calculating $V_{\nu}(R)$ by solving Eq. (31) one should use the full effective charge (20), not the first term of its expansion in $h$ (25). Even though the difference is of the same order $O\left(h^{2}\right)$, it cannot be neglected because this may modify the asymptotic values of $V_{\nu}(R)$ for $R \rightarrow \infty$, which must coincide with the threshold energies (21). But this does not happen for systems under present consideration: both formulas (27) are consistent with Eq. (28).

\section{Transition points}

The points where the leading order approximation breaks down are called the transition points. There are two types of transition points in our problem: turning points $R_{t}^{\nu}$, where $K_{\nu}(R)=0$ and the primitive SC solutions (44) diverge, and crossing points $R_{c}^{\nu \mu}$, where $V_{\nu}(R)=V_{\mu}(R)$ and the first order corrections (42) diverge. Here we discuss the distribution of transition points in the complex $R$ plane needed for constructing a global SC solution.

\section{Crossing points}

Equation (31) for our problem explicitly reads

$$
\left[-\frac{1}{2} \frac{d^{2}}{d \phi^{2}}+R C(\phi)-U\right] \Phi(\phi)=0 .
$$

The primitive SC solutions (44) must satisfy Eqs. (11) (where $\gamma_{3}$ should be replaced by $\pi / 2$, because of scaling), which leads to the boundary conditions

$$
\Phi(0)=\Phi(\pi / 2)=0 .
$$

The solutions to this eigenvalue problem $U(R)$ and $\Phi(\phi ; R)$ are multivalued analytic functions of $R$. The different branches of these functions obtained by the analytic continuation from the real axis perpendicular to it will be denoted by $U_{\nu}(R)$ and $\Phi_{\nu}(\phi ; R)$, as in Eq. (31). The crossing points $R_{c}^{\nu \mu}$ are defined by the condition $U_{\nu}(R)=U_{\mu}(R)$ and are generally branch points of the functions $U(R)$ and $\Phi(\phi ; R)$. Because the eigenvalues of Eqs. (47) cannot coincide for real values of $R$, all the crossing points have nonzero imaginary parts and appear in complex conjugate pairs. Our definition of $U_{\nu}(R)$ and $\Phi_{\nu}(\phi ; R)$ assumes that branch cuts are made perpendicular to the real axis upwards (downwards) from the branch points located in the upper (lower) half of the complex plane. A complex plane with cuts appropriate for the given $\nu$ will be called the sheet $\nu$. Gluing all the sheets along the corresponding cuts, one obtains a Riemann surface upon which $U(R)$ [but not $\Phi(\phi ; R)$, see the discussion below] becomes single valued. This surface provides a natural arena for studying dynamics in the SC approximation [34].

A practical way to solve Eqs. (47a) and (47b) for real as well as complex values of $R$ is to reduce the problem to an algebraic form. Given a suitable orthonormal basis $\pi_{i}(\phi), i$ $=1,2, \ldots, N$, that becomes complete in the interval $0 \leqslant \phi$ $\leqslant \pi / 2$ as $N \rightarrow \infty$ and satisfies $\pi_{i}(0)=\pi_{i}(\pi / 2)=0$, the solutions to Eqs. (47a) and (47b) can be sought in the form

$$
\Phi(\phi)=\sum_{i=1}^{N} c_{i} \pi_{i}(\phi) .
$$

Substituting this expansion into Eqs. (47a) and (47b), one obtains an algebraic eigenvalue problem,

$$
\left[\frac{1}{2} \Lambda^{2}+R \mathbf{C}-U \mathbf{I}\right] \mathbf{c}=\mathbf{0}
$$

where matrices $\boldsymbol{\Lambda}^{2}$ and $\mathbf{C}$ represent the grand angular momentum operator squared $\Lambda^{2}=-d^{2} / d \phi^{2}$ and the effective charge $C(\phi)$, $\mathbf{c}$ is the vector of coefficients in Eq. (48), and $\mathbf{I}$ is a unit matrix. Equation (49) can be solved using standard linear algebra routines. The region of convergence of the results in the complex $R$ plane depends on the basis. In the present calculations we use the same discrete variable representation basis constructed from the Jacobi polynomials $P_{n}^{(1,1)}(x)$ as in the program СТВC, for more details see Ref. [19]. This basis is well adapted to the Coulomb singularities of $C(\phi)$ at $\phi=0$ and $\pi / 2$ and provides a high rate of convergence as $N$ grows. Besides, it permits one to easily implement the symmetry boundary conditions (12) (where, again, $\gamma_{3}$ should be changed to $\pi / 2$ ) in the symmetric case, so the different permutation symmetries $\sigma= \pm$ in case A can be treated separately. The matrices $\boldsymbol{\Lambda}^{2}$ and $\mathbf{C}$ in this basis are real and symmetric, therefore for any complex $R$ the eigenvectors corresponding to different eigenvalues $U_{\nu}$ and $U_{\mu}$ are orthogonal, $\mathbf{c}_{\nu}^{T} \mathbf{c}_{\mu}=0$, without complex conjugation.

The eigenvalues of Eq. (49) are algebraic functions of $R$. It is natural to expect that in the general case all their branch points are of square root type. Let $R_{c}$ be a square root branch point, i.e., matrix $\mathcal{U}\left(R_{c}\right)=\frac{1}{2} \Lambda^{2}+R_{c} \mathbf{C}$ has a pair of equal eigenvalues $U_{c}$. By an orthogonal transformation of the basis this matrix can be reduced to the diagonal form except for a $2 \times 2$ block corresponding to the degenerate subspace. Let vectors $\mathbf{e}_{1}$ and $\mathbf{e}_{2}, \mathbf{e}_{i}^{T} \mathbf{e}_{j}=\delta_{i j}$, form an orthonormal basis in the degenerate subspace. In any such basis, the nondiagonal block of $\mathcal{U}\left(R_{c}\right)$ is symmetric and has the form

$$
\left(\begin{array}{cc}
U_{c}+u & i u \\
i u & U_{c}-u
\end{array}\right)
$$

This matrix has two equal eigenvalues $U_{c}$, but only one eigenvector $(1, i)^{T}$ with zero norm. Introducing a new basis $\boldsymbol{\varepsilon}_{1}=u^{1 / 2}\left(\mathbf{e}_{1}+i \mathbf{e}_{2}\right), \boldsymbol{\varepsilon}_{2}=u^{-1 / 2}\left(\mathbf{e}_{1}-i \mathbf{e}_{2}\right) / 2$ with rather unusual orthogonality properties $\boldsymbol{\varepsilon}_{1}^{T} \boldsymbol{\varepsilon}_{1}=\boldsymbol{\varepsilon}_{2}^{T} \boldsymbol{\varepsilon}_{2}=0$ and $\boldsymbol{\varepsilon}_{1}^{T} \boldsymbol{\varepsilon}_{2}=1$, matrix (50) can be transformed to the normal Jordan form, however, in practical calculations it is more convenient to work with an orthonormal basis. For any $R \neq R_{c}$ in the vicinity of the branch point, Eq. (49) has $N$ different eigenvalues $U_{\nu}(R)$ and corresponding orthogonal eigenvectors $\mathbf{c}_{\nu}(R)$ that can be normalized by $\mathbf{c}_{\nu}^{T}(R) \mathbf{c}_{\mu}(R)=\delta_{\nu \mu}$. As $R \rightarrow R_{c}$, the pair of solutions that become degenerate at $R=R_{c}$ behave as

$$
U_{ \pm}(R)=U_{c} \pm v z^{1 / 2}+O(z)
$$




$$
\mathbf{c}_{ \pm}(R)=\frac{s_{ \pm}}{\sqrt{2}}\left[\widetilde{z}^{-1 / 4}\left(\mathbf{e}_{1}+i \mathbf{e}_{2}\right) \pm \widetilde{z}^{1 / 4} \mathbf{e}_{1}+O\left(z^{3 / 4}\right)\right],
$$

where $z=R-R_{c}, \tilde{z}=z v^{2} / u^{2}, s_{+}=1, s_{-}=i$, and $v$ is another parameter characterizing the branch point. The other solutions stay constant within the specified accuracy. As can be seen from Eqs. (51), when $R$ circles four times around $R_{c}$ counterclockwise, the solutions $\left(U_{ \pm}, \mathbf{c}_{ \pm}\right)$transform into each other according to

$$
\begin{aligned}
\left(U_{ \pm}, \mathbf{c}_{ \pm}\right) & \rightarrow\left(U_{\mp}, \mp \mathbf{c}_{\mp}\right) \rightarrow\left(U_{ \pm},-\mathbf{c}_{ \pm}\right) \rightarrow\left(U_{\mp}, \pm \mathbf{c}_{\mp}\right) \\
& \rightarrow\left(U_{ \pm}, \mathbf{c}_{ \pm}\right) .
\end{aligned}
$$

Thus $\Phi(\phi ; R)$ is a double valued function on the Riemann surface of $U(R)$ [35]. Using Eq. (51b), from Eqs. (33) we obtain

$$
\begin{aligned}
& P_{+-}(R)=-P_{-+}(R)=\frac{-i}{4 z}+O\left(z^{-1 / 2}\right), \\
& Q_{++}(R)=Q_{--}(R)=\frac{1}{16 z^{2}}+O\left(z^{-3 / 2}\right), \\
& Q_{+-}(R)=-Q_{-+}(R)=\frac{i}{4 z^{2}}+O\left(z^{-3 / 2}\right) .
\end{aligned}
$$

The divergence of nonadiabatic couplings at crossing points again indicates that the primitive SC solutions (44) become invalid there.

The numerical procedure of finding branch points is a technical issue, however, this issue is very important for implementing the SC theory. We are not aware of any standard approach to this problem, so let us describe briefly our own one. In principle, branch points could be found covering the region of interest in the complex $R$ plane by a sufficiently dense mesh and then making the mesh finer near the points where a pair of eigenvalues seems to coalesce. However, besides being very time consuming such a straightforward approach does not guarantee that the results are really branch points and that all the branch points in the region are found. Our approach is much more efficient and reliable. It is based on three major elements. The first and the most important one is the procedure of numerical "analytic continuation." Given the solutions to Eq. (49) at two points, $\left\{U_{\nu}(R), \mathbf{c}_{\nu}(R)\right\}$ and $\left\{U_{\nu}(R+\Delta R), \mathbf{c}_{\nu}(R+\Delta R)\right\}, \nu=1, \ldots, N$, how to establish the correspondence between the two sets dictated by the analyticity of their dependence on $R$ ? We found that the eigenvectors provide a more sensitive criterion than the eigenvalues. For each solution at $R$, we look for a solution at $R$ $+\Delta R$ satisfying the condition $\left|\mathbf{c}_{\nu}^{T}(R) \mathbf{c}_{\mu}(R+\Delta R)-1\right|<\epsilon$, where $\epsilon$ is a given small number typically of the order of $10^{-3}$. If there is no such solution or there is more than onethe interval $\Delta R$ is divided into two parts, and the procedure is repeated. In this way the solutions of Eq. (49) can be "analytically continued" along any path in the complex $R$ plane. The second element permits one to determine whether there are branch points in the given region. To this end, the solutions are analytically continued around the region along its boundary, and after returning to the initial point the new

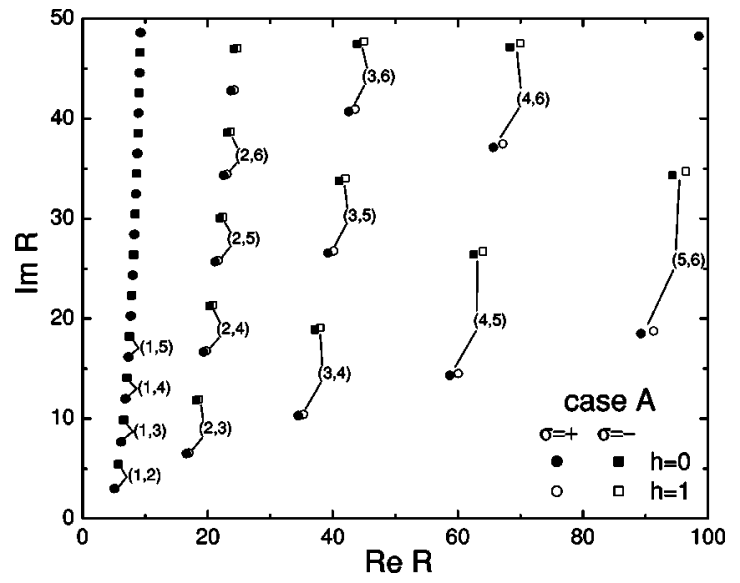

FIG. 3. The distribution of crossing points for two extreme values of $h$ and two permutation symmetries $\sigma= \pm$ in case A. Some of the crossing points connecting low-lying states $\nu$ and $\mu$ are labeled by $(\nu, \mu)$; for more details on their classification see the text.

eigenvalues are compared with the old ones. A difference in the orders of the two sets indicates the presence of branch points. Of course, it is possible that there are branch points (and in this case more than one) even if the two sets coincide. To minimize the risk of missing something, in our calculations we divide the whole region of interest into a number of rectangles and apply the above procedure to each of them. The third element is finding a branch point connecting the given pair of solutions and located in the given rectangle. This can be done iteratively using Eq. (51a). The procedure usually converges in a few iterations; if it does not, the rectangle is divided into four parts, and each of them is considered again. This approach is rather universal and can be applied to any eigenvalue problem of the type (49). The only parameter one has to choose based on the a priori information is the initial size of the rectangles, and this can be easily done having a little experience in the problem.

The results of our calculations for $C(\phi)$ given by Eq. (27a) are presented in Figs. 3 and 4, see also Table I. Only crossing points located in the first quadrant are shown; it should be remembered that their complex conjugate partners

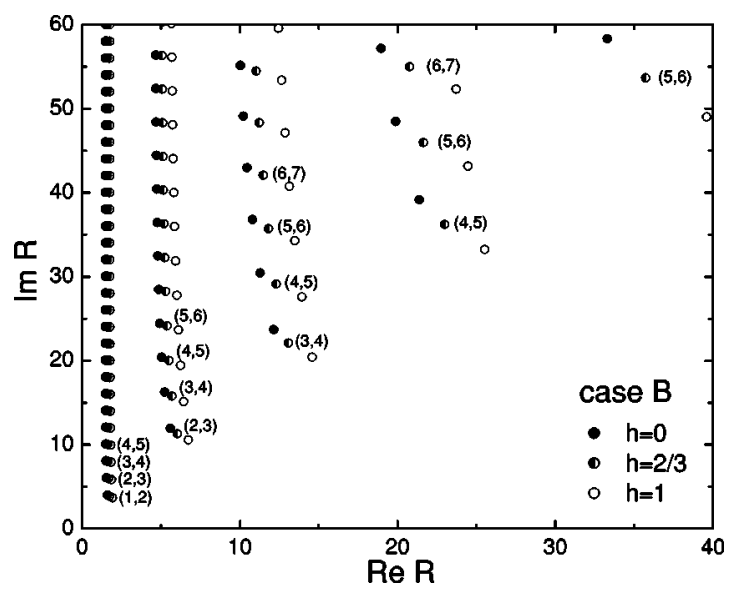

FIG. 4. Same as in Fig. 3, but for three representative values of $h$ in case B. 
TABLE I. Hidden crossings of the main series in systems with $h=2 / 3$. QM, the quantum-mechanical results discussed in Sec. IV C 1 ; USC, the uniform semiclassical results obtained from Eqs. (81) and (82) by solving Eqs. (88a) and (88b); and AS, the asymptotic results for $\nu \rightarrow \infty$ obtained from Eqs. (89) and (95).

\begin{tabular}{|c|c|c|c|c|c|c|}
\hline \multirow[b]{2}{*}{$\nu$} & \multicolumn{3}{|c|}{$R_{c}^{\nu, \nu+1}$} & \multicolumn{3}{|c|}{$U_{c}^{\nu, \nu+1}$} \\
\hline & QM & USC & AS & QM & USC & AS \\
\hline \multicolumn{7}{|c|}{ Case A, $\sigma=+$} \\
\hline 1 & $(5.10223,2.99735)$ & $(5.18811,2.99883)$ & & $(-7.36633,-12.7779)$ & $(-7.87989,-13.0372)$ & \\
\hline 2 & $(16.7825,6.52054)$ & $(16.8699,6.52138)$ & $(17.77,3.257)$ & $(-30.6647,-23.6962)$ & $(-31.1727,-23.8313)$ & $(-30.52,-33.90)$ \\
\hline 3 & $(34.8879,10.3552)$ & $(34.9757,10.3557)$ & $(35.85,6.891)$ & $(-66.8193,-35.1010)$ & $(-67.3198,-35.1889)$ & $(-66.56,-40.53)$ \\
\hline 4 & $(59.3827,14.4058)$ & $(59.4707,14.4062)$ & $(60.33,10.60)$ & $(-115.764,-46.8609)$ & $(-116.263,-46.9289)$ & $(-115.5,-50.43)$ \\
\hline 5 & $(90.2525,18.6211)$ & $(90.3405,18.6214)$ & $(91.20,14.44)$ & $(-177.466,-58.8979)$ & $(-177.960,-58.9555)$ & $(-177.3,-61.34)$ \\
\hline 10 & $(340.054,41.3333)$ & $(340.142,41.3334)$ & $(341.0,35.26)$ & $(-676.935,-121.801)$ & $(-677.429,-121.943)$ & $(-676.7,-121.1)$ \\
\hline \multicolumn{7}{|c|}{ Case A, $\sigma=-$} \\
\hline 1 & $(5.71804,5.43126)$ & $(5.80487,5.43292)$ & & $(-0.23921,-27.1943)$ & $(-0.80527,-27.7132)$ & \\
\hline 2 & $(18.3467,11.8710)$ & $(18.4347,11.8723)$ & $(21.03,8.635)$ & $(-24.7948,-49.5047)$ & $(-25.3279,-49.7767)$ & $(-21.91,-66.39)$ \\
\hline 3 & $(37.5563,18.9655)$ & $(37.6444,18.9665)$ & $(40.24,15.84)$ & $(-62.6801,-72.8404)$ & $(-63.1891,-73.0321)$ & $(-60.27,-82.98)$ \\
\hline 4 & $(63.2459,26.5367)$ & $(63.3341,26.5375)$ & $(65.94,23.32)$ & $(-113.637,-96.9847)$ & $(-114.144,-97.1352)$ & $(-111.3,-104.8)$ \\
\hline 5 & $(95.3708,34.4822)$ & $(95.4590,34.4828)$ & $(98.09,31.11)$ & $(-177.536,-121.785)$ & $(-178.039,-121.905)$ & $(-175.2,-128.3)$ \\
\hline 10 & $(351.928,78.0507)$ & $(352.016,78.0510)$ & $(354.8,73.78)$ & $(-689.443,-252.559)$ & $(-689.977,-252.789)$ & $(-686.9,-256.5)$ \\
\hline \multicolumn{7}{|c|}{ Case B } \\
\hline 1 & $(1.72506,3.86073)$ & $(1.69882,3.84472)$ & & $(6.86464,-5.74570)$ & $(6.80768,-5.69015)$ & \\
\hline 2 & $(6.02587,11.2914)$ & $(6.00036,11.2708)$ & $(8.241,10.30)$ & $(14.4291,-15.9494)$ & $(14.3763,-15.8970)$ & $(19.82,-21.90)$ \\
\hline 3 & $(13.0900,22.0908)$ & $(13.0647,22.0682)$ & $(15.44,21.10)$ & $(24.1484,-31.3548)$ & $(24.0940,-31.3030)$ & $(27.65,-35.82)$ \\
\hline 4 & $(22.9877,36.2251)$ & $(22.9626,36.2015)$ & $(25.55,35.15)$ & $(35.9816,-52.0207)$ & $(35.9267,-51.9683)$ & $(38.78,-56.21)$ \\
\hline 5 & $(35.7397,53.6699)$ & $(35.7147,53.6458)$ & $(38.52,52.50)$ & $(49.8953,-77.9415)$ & $(49.8401,-77.8905)$ & $(52.29,-82.14)$ \\
\hline 10 & $(142.668,190.122)$ & $(142.644,190.097)$ & $(146.6,188.4)$ & $(150.119,-286.438)$ & $(150.025,-286.446)$ & $(151.5,-291.5)$ \\
\hline
\end{tabular}

lie in the fourth quadrant. We found that in both cases A and $\mathrm{B}$ there are crossing points only of the square root type. They form a regular two-dimensional pattern that can be roughly described as a deformed rectangular lattice. The lowest row of the lattice will be called the main series, the higher rows will be called the secondary series. The crossing points of the main series have the same classification in cases $\mathrm{A}$ and $\mathrm{B}$, namely, they connect consecutively states $\nu$ and $\nu+1$, starting with $\nu=1$ for the leftmost point. The vertical columns of the lattice have slight positive (negative) slopes in case A (B), which causes an essential difference in the classification of the other crossing points. If $\nu=1,2, \ldots$ enumerates columns and $n=0,1, \ldots$ enumerates rows of the lattice, then the crossing point with the given values of $\nu$ and $n$ in case A (B) connects states $\nu(\nu+n)$ and $\nu+n+1$. This means that in case A any pair of states $\nu$ and $\mu$ are connected by a single crossing point, while in case B only neighboring states $\nu$ and $\nu$ +1 are connected and there are $\nu$ crossing points joining them. In both cases, the Riemann surface of the adiabatic eigenvalue $U(R)$ is singly connected. Note that in case A crossing points for the different permutation symmetries $\sigma$ $= \pm$ actually form a single regular pattern. This is not surprising because although the Riemann surfaces for the two symmetries are disconnected, they correspond to eigenvalues of the same adiabatic Hamiltonian. Figures 3 and 4 also illustrate the dependence of the distribution of crossing points on the asymptotic parameter $h$ caused by the dependence on $h$ of the effective charge (27a). As one could expect from Fig. 2 , this dependence is rather weak. More specifically, the positions of crossing points in columns with low $\nu$ depend on $h$ more weakly than in columns with higher $\nu$, and this dependence in case $\mathrm{B}$ is more pronounced than in case $\mathrm{A}$.

It is instructive to see how crossing points reveal themselves in the adiabatic eigenvalues $U_{\nu}(R)$ and matrix elements of nonadiabatic couplings $P_{\nu \mu}(R)$ on the real axis. This is illustrated in Figs. 5 and 6 for an intermediate value of $h=2 / 3$; the dependence on $h$ is weak, so the situation for other values of $h$ is similar. As follows from Eqs. (27a) and (47a) and (47b),

$$
U_{\nu}(R=0)= \begin{cases}2(2 \nu-1)^{2}, & \text { case } \mathrm{A}, \sigma=+, \\ 8 \nu^{2}, & \text { case A, } \sigma=-, \\ 2 \nu^{2}, & \text { case B }\end{cases}
$$

and in all the cases

$$
U_{\nu}(R \rightarrow \infty)=E_{\nu} R^{2},
$$

thus $U_{\nu}(R)$ change sign as $R$ grows from 0 to $\infty$. The middle panels in the figures show the eigenvalues $U_{\nu}(R)$ in the domain where they are positive. In the domain where $U_{\nu}(R)$ are negative, it is more convenient to consider the effective quantum numbers (32b) shown in the lower panels. The up- 


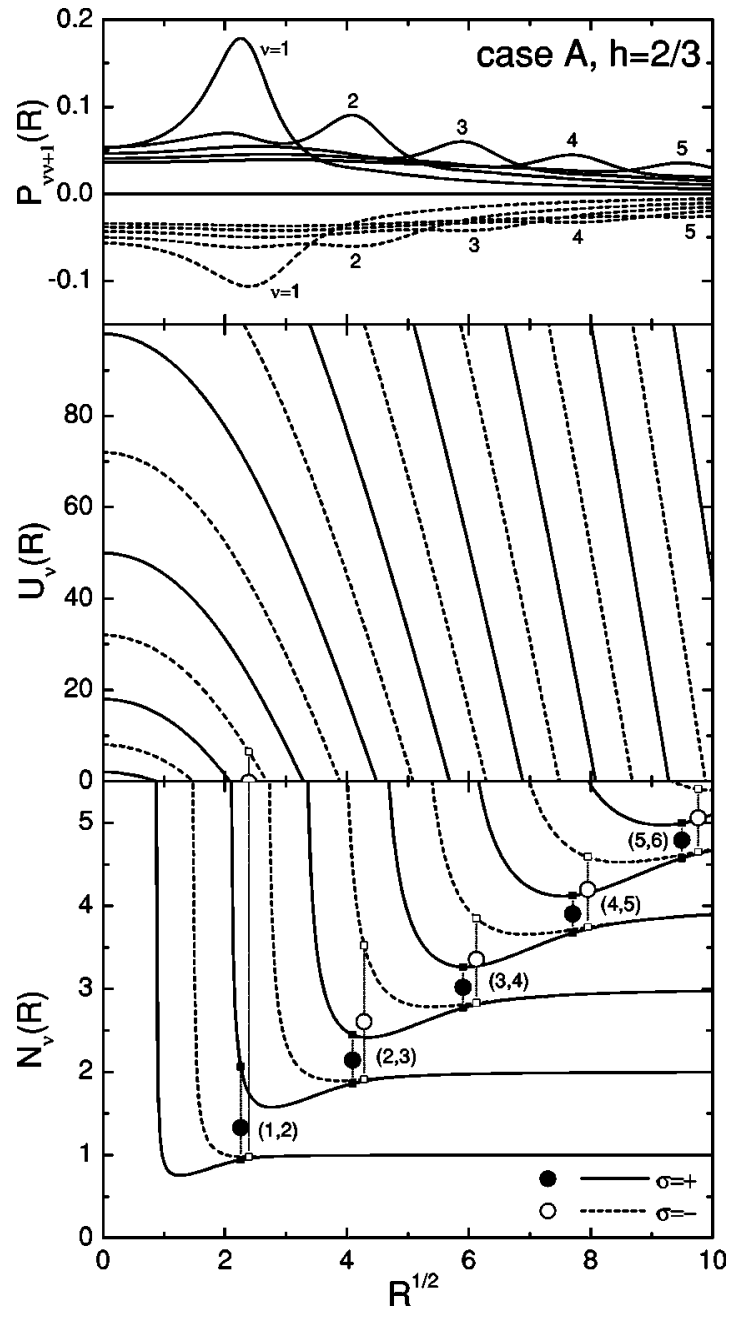

FIG. 5. The adiabatic eigenvalues (shown in two different formats in the middle and lower panels) and nonadiabatic couplings (33a) between neighboring states (in the upper panel) for $h=2 / 3$ and two permutation symmetries $\sigma= \pm$ in case A. The signs of $P_{\nu \nu+1}(R)$ are changed to make these functions positive (negative) for $\sigma=+(\sigma=-)$. Circles show the projections of crossing points of the main series. Vertical dotted lines with small squares at the ends indicate states connected by the crossing points with the same abscissas.

per panels show the nonadiabatic couplings (33a) between neighboring states. The projections of crossing points of the main series on the plane of real $R$ and $U$ or $N$ are also shown. The first feature to be noticed from these results is that in case A crossing points of the main series lie in the negative $U$ domain, while in case B they lie in the positive $U$ domain, see also Table I. Second, in case A curves $N_{\nu}(R)$ exhibit a clear creaselike structure along the main series of crossing points, while in case B there is not any visible structure in the adiabatic eigenvalues and in this sense crossing points lie in quite unexpected places. The third feature concerns the nonadiabatic couplings. As can be seen from Eq. (53a), $P_{\nu \mu}(R)$ diverge at crossing points. Each pair of complex conjugate crossing points $R_{c}$ and $R_{c}^{*}$ produces a bell-shaped contribution to the corresponding curve $P_{\nu \mu}(R)$ on the real axis with the maximum near $R=\operatorname{Re} R_{c}$. In case $\mathrm{A}$, there is only

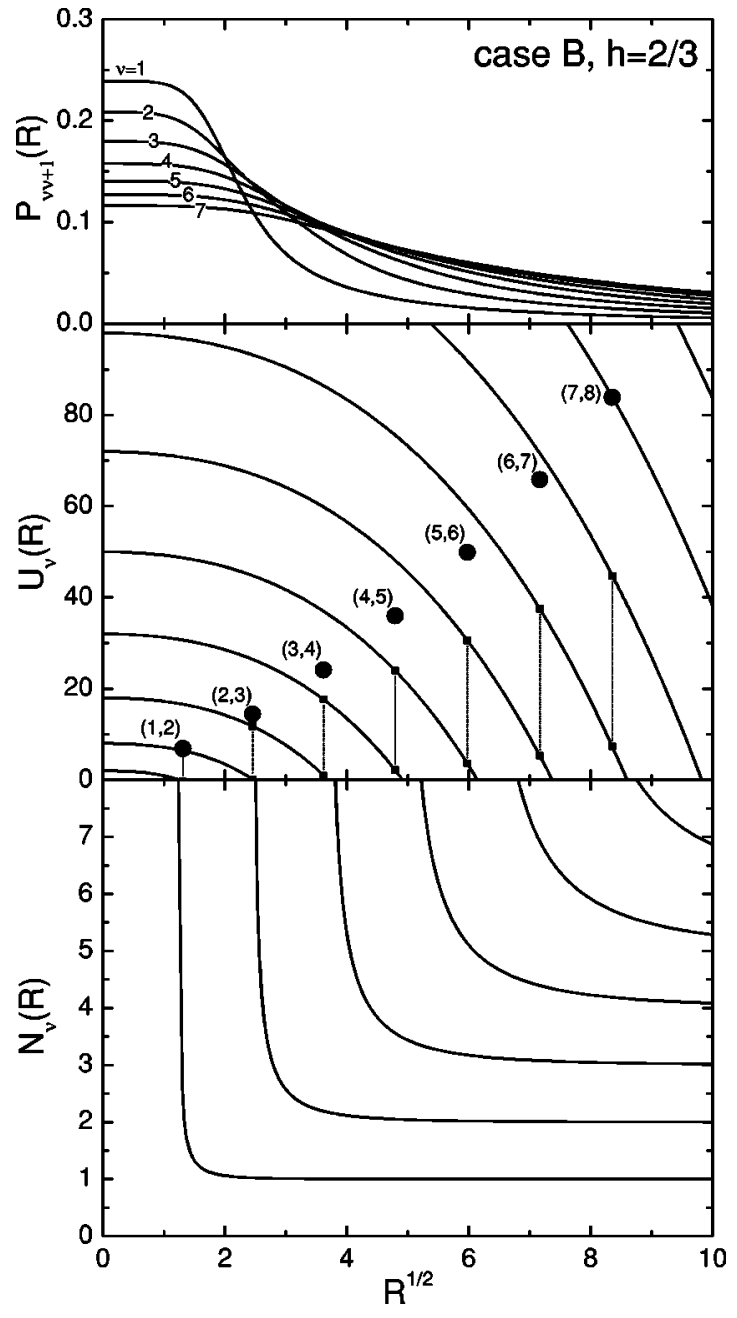

FIG. 6. Same as in Fig. 5, but in case B. Note that in this case the projections of crossing points lie higher than the eigenvalues for states connected by them taken on the real axis.

one such pair connecting states $\nu$ and $\nu+1$, and the absolute values of $P_{\nu \nu+1}(R)$ indeed have maxima at the expected positions, see Fig. 5. In case B, there are $\nu$ crossing points connecting states $\nu$ and $\nu+1$. Their joint contributions produce a shoulder in the curve $P_{\nu \nu+1}(R)$ that extends from $R$ $=0$ to the rightmost crossing point for the given $\nu$, which is the one belonging to the main series, see Fig. 6 .

\section{Turning points}

The turning points $R_{t}^{\nu}$ are defined by the condition $K_{\nu}(R)=0$, i.e., $U_{\nu}(R)=E R^{2}$. The problem of finding turning points can be reduced to an eigenvalue problem. Indeed, substituting $U=E R^{2}$ into Eq. (47a), in the algebraic form similar to Eq. (49), one obtains

$$
\left[\frac{1}{2} \boldsymbol{\Lambda}^{2}+R \mathbf{C}-E R^{2} \mathbf{I}\right] \mathbf{c}=\mathbf{0} .
$$

This is a quadratic eigenvalue problem in $R$. It can be linearized by doubling its dimension (a similar technique is used in the theory of Siegert states [36]), 


$$
\left[\left(\begin{array}{cc}
\mathbf{0} & \mathbf{I} \\
\frac{1}{2} \boldsymbol{\Lambda}^{2} & \mathbf{C}
\end{array}\right)-R\left(\begin{array}{cc}
\mathbf{I} & \mathbf{0} \\
\mathbf{0} & E \mathbf{I}
\end{array}\right)\right]\left(\begin{array}{c}
\mathbf{c} \\
\widetilde{\mathbf{c}}
\end{array}\right)=\mathbf{0}
$$

where $\widetilde{\mathbf{c}}=R \mathbf{c}$, and then solved by standard routines. Turning points are given by the eigenvalues to this equation for a sufficiently large basis. Note that the possibility to linearize Eq. (56) rests on a very simple (linear) dependence of the adiabatic Hamiltonian (30) on $R$, which is a common property for Coulomb systems treated in hyperspherical coordinates.

In contrast to crossing points that are completely determined by the adiabatic Hamiltonian, turning points depend also on the energy $E$. They can be divided into two groups, real and complex; the latter appear in complex conjugate pairs. The number and positions of real turning points $R_{t}^{\nu}$ for the given $\nu$ are determined by the behavior of the adiabatic potentials $V_{\nu}(R)$ on the real axis. As follows from Eqs. (32a), (54), and (55), functions $V_{\nu}(R)$ diverge $\propto R^{-2}$ with a positive coefficient as $R \rightarrow 0$ and asymptotically approach the negative values $E_{\nu}$ as $R \rightarrow \infty$. For the systems under consideration, they have one minimum for both permutation symmetries $\sigma= \pm$ in case A and are monotonic in case B. Let

$$
V_{\nu}^{\min }=\min \left[V_{\nu}(R)\right],
$$

so that $V_{\nu}^{\min }<E_{\nu}$ in case $\mathrm{A}$ and $V_{\nu}^{\mathrm{min}}=E_{\nu}$ in case $\mathrm{B}$. Then there are two (one) real turning points for the given $\nu$ in the energy interval $V_{\nu}^{\mathrm{min}}<E<E_{\nu}\left(E_{\nu}<E\right)$. The positions of complex turning points are less intuitive. As $E$ varies, they move along certain trajectories in the complex plane that can be calculated by solving Eq. (57). We found that there are no complex turning points in the domain $\operatorname{Re} R>0$ for $E>0$. The trajectories of complex turning points for $E<0$ are shown in Figs. 7 and 8. On each sheet $\nu$ of the Riemann surface of $U(R)$, there is one trajectory starting from $R=0$ for $E=-\infty$ tangentially to the imaginary axis. Its further behavior in cases $\mathrm{A}$ and $\mathrm{B}$ is different. In case $\mathrm{A}$, the corresponding turning point moves along an arc as $E$ grows, temporarily leaving sheet $\nu$ to make a pirouette around crossing points it encounters, and returns to the real axis on the same sheet $\nu$ at the point where $V_{\nu}(R)$ has the minimum. Here, it and its complex conjugate partner coalesce and turn into a pair of real turning points as $E$ grows further. In case $\mathrm{B}$, the trajectory never returns to the real axis and goes to complex infinity as $E \rightarrow-0$. The trajectories of complex turning points can be viewed also from a different side-they show the lines in the complex $R$ plane along which the adiabatic potentials $V_{\nu}(R)$ are pure real.

\section{Connection formulas}

In the leading order approximation, a general solution to Eq. (36) is given by a linear combination of the primitive SC solutions (44),

$$
\psi=\sum_{\nu}\left(a_{\nu}^{+} \psi_{\nu}^{+}+a_{\nu}^{-} \psi_{\nu}^{-}\right)
$$

However, because of the Stokes phenomenon this representation is valid only locally. Namely, if Eq. (59) gives a solu-

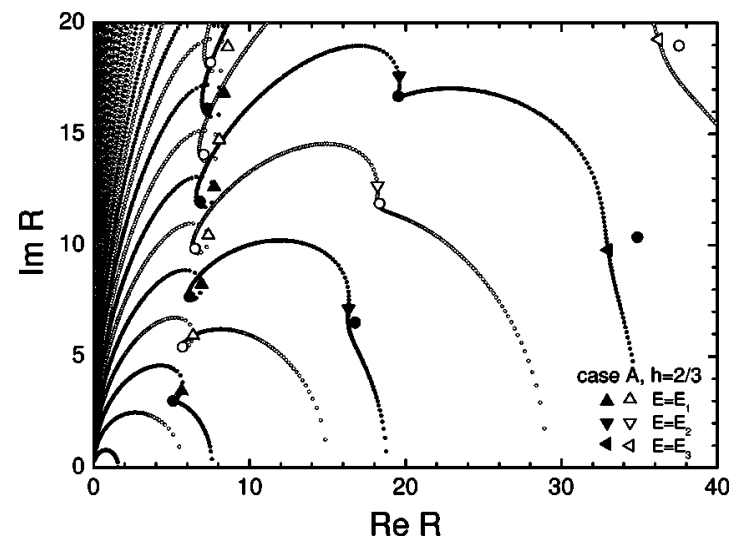

FIG. 7. Trajectories of complex turning points for $h=2 / 3$ in case A. Small circles, turning points for $E<0$; large circles, crossing points, see Fig. 3; triangles, turning points for $E=E_{\nu}$, see Eq. (15). Closed (open) symbols correspond to $\sigma=+(\sigma=-)$.

tion to Eq. (36) in one region of complex $R$ plane bounded by the Stokes lines emanating from transition (crossing and turning) points, then in another region the same solution is given by Eq. (59) with a generally different set of coefficients $a_{\nu}^{ \pm}$. To construct a global solution one needs to know a relation between the coefficients, which is called the connection formulas. In the leading order approximation it is assumed that all actions for the motion in $R$ involved in the formulation become infinitely large in the limit $h \rightarrow 0$, i.e., there are no coalescing transition points. Then the connection formulas can be easily derived using the method of Zwaan [37]. An introduction to this method can be found in [38], its recent developments are discussed in [39]. Stueckelberg [21] was the first who applied it to the problem of nonadiabatic transitions and obtained the key result to be used below; some inaccuracies of Stueckelberg's analysis were clarified by Crothers [40], see also Appendix A in [3]. More rigorous discussion of connection formulas can be found in [41,42]. Because all these issues are well documented in the literature, here we only give some details specific to our problem and summarize the final formulas used in the calculations.

We call the solutions $\psi_{\nu}^{ \pm}$whose coefficients $a_{\nu}^{ \pm}$in Eq. (59) may experience a discontinuity on crossing the given Stokes line coupled by the corresponding transition point. Each tran-

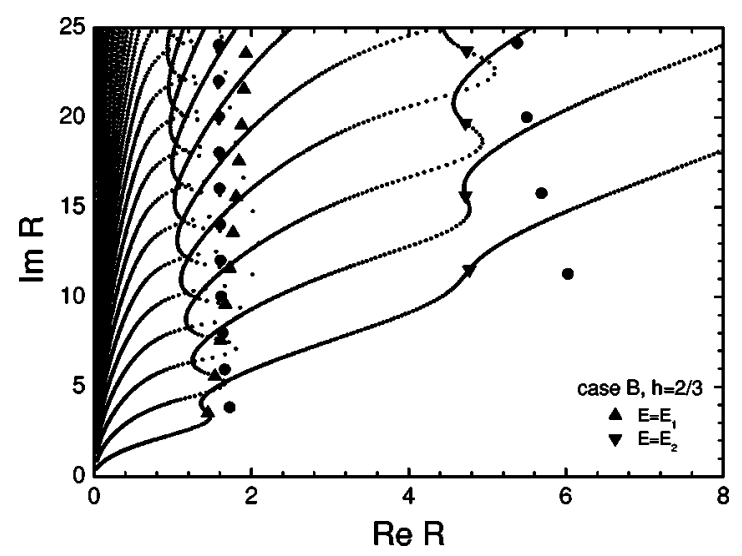

FIG. 8. Same as in Fig. 7, but in case B. See also Fig. 4. 
(a)

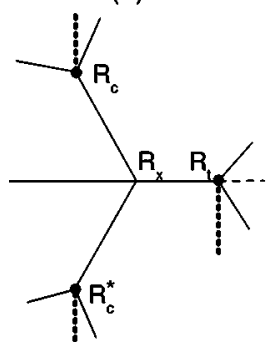

(c)

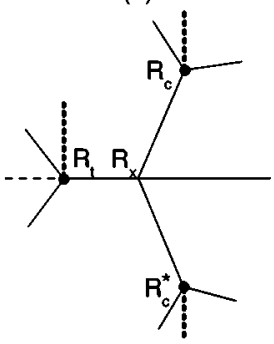

FIG. 9. Schematic representation of the Stokes lines (solid lines) and branch cuts (thick dashed lines) near a pair of complex conjugate crossing points $R_{c}$ and $R_{c}^{*}$. The adiabatic momentum in the upper of the two crossing states at the point $R_{x}$ is real (b) and imaginary in the presence of a left-hand (a) and right-hand (c) turning point $R_{t}$.

sition point couples a pair of solutions that can be obtained from each other by the analytic continuation around it. Turning points $R_{t}^{\nu}$ couple $\psi_{\nu}^{+}$and $\psi_{\nu}^{-}$, which leads to reflection. The reflection coefficient at real turning points for waves propagating along the real axis is $O\left(h^{0}\right)$, so they surely must be taken into account in constructing a global solution. Complex turning points cause reflection with an exponentially small coefficient, similarly to the above-barrier reflection; in the leading order approximation their effect should be neglected because we have already neglected much larger contributions $O(h)$ to the phase in Eq. (44). Crossing points $R_{c}^{\nu \mu}$ couple each of $\psi_{\nu}^{ \pm}$with one of $\psi_{\mu}^{ \pm}$, which leads to nonadiabatic transitions. Which one of these solutions is coupled with which depends on whether $K_{\nu}(R)=K_{\mu}(R)$ or $K_{\nu}(R)=$ $-K_{\mu}(R)$ at $R=R_{c}^{\nu \mu}$ and is determined by the branch of $K_{\nu}(R)$ chosen and its behavior under the analytic continuation around $R_{c}^{\nu \mu}$. Let us discuss this issue.

A channel $\nu$ is called open (closed) for the given energy if $E>E_{\nu}\left(E<E_{\nu}\right)$; it will be called active if $E>V_{\nu}^{\mathrm{min}}$. Only active channels should be considered in the leading order approximation. The adiabatic momentum $K_{\nu}(R)$ is a double valued function on sheet $\nu$. To make it single valued, we cut each sheet $\nu$ from the left-hand (right-hand) real turning points $R_{t}^{\nu}$ located on it downwards (upwards), see Fig. 9; one should also add appropriate cuts starting at complex turning points, but they are immaterial for the following discussion. We choose the branch in Eq. (32c) such that $K_{\nu}(R)$ is positive real (imaginary) in classically allowed (forbidden) intervals of the real axis. Then $\psi_{\nu}^{+}\left(\psi_{\nu}^{-}\right)$represent waves going to the right (left) in the allowed intervals and exponentially decaying (growing) in the forbidden ones. Let $R_{c}^{\nu \mu}$ be a crossing point connecting two active channels (we shall always assume in this notation that $\nu<\mu$ ), and $R_{x}$ be a real point lying near $\operatorname{Re}\left(R_{c}^{\nu \mu}\right)$. Suppose both $K_{\nu}\left(R_{x}\right)$ and $K_{\mu}\left(R_{x}\right)$ are real, see Fig. 9(b). Consider the analytic continuation of $K_{\nu}(R)$ along a path on the Riemann surface of $U(R)$ starting at $R_{x}$ on sheet $\nu$, going upwards, around $R_{c}^{\nu \mu}$, and returning to the real axis at $R_{x}$ on sheet $\mu$. As a result we can obtain either $K_{\mu}\left(R_{x}\right)$ or $-K_{\mu}\left(R_{x}\right)$, depending on whether $\operatorname{Re}\left[K_{\nu}(R)\right]$ changes its sign on the way, which occurs if the path crosses a line where $V_{\nu}(R)$ is pure real and larger than $E$. But as follows from the results of Sec. IV C 2, see Figs. 7 and 8, this never happens for active channels. Thus the result is $K_{\mu}\left(R_{x}\right)$, which means that $K_{\nu}\left(R_{c}^{\nu \mu}\right)=K_{\mu}\left(R_{c}^{\nu \mu}\right)$, i.e., the crossing point $R_{c}^{\nu \mu}$ couples $\psi_{\nu}^{+}$with $\psi_{\mu}^{+}$and $\psi_{\nu}^{-}$with $\psi_{\mu}^{-}$. A similar consideration in the case if $K_{\mu}\left(R_{x}\right)$ is imaginary shows that this point (a) couples the same pairs of solutions in the presence of a left-hand turning point $R_{t}^{\mu}$, see Fig. 9(a), because in this case again $K_{\nu}\left(R_{c}^{\nu \mu}\right)=K_{\mu}\left(R_{c}^{\nu \mu}\right)$; (b) couples the opposite pairs, i.e., $\psi_{\nu}^{+}$ with $\psi_{\mu}^{-}$and $\psi_{\nu}^{-}$with $\psi_{\mu}^{+}$, in the presence of a right-hand turning point $R_{t}^{\mu}$, see Fig. 9(c), because in this case $K_{\nu}\left(R_{c}^{\nu \mu}\right)=-K_{\mu}\left(R_{c}^{\nu \mu}\right)$. It can be also shown that the complex conjugate crossing point $\left(R_{c}^{\nu \mu}\right) *$ couples the same pairs of solutions as $R_{c}^{\nu \mu}$ if $K_{\mu}\left(R_{x}\right)$ is real, and the opposite pairs if $K_{\mu}\left(R_{x}\right)$ is imaginary. We wish to emphasize that the simplicity of this coupling scheme results from the fact that it is not affected by complex turning points, which is a property of our problem.

Let us introduce some notation. The action for the motion in $R$ in state $\nu$ is

$$
S_{\nu}\left[R^{\prime}, R^{\prime \prime}\right]=h^{-1} \int_{R^{\prime}}^{R^{\prime \prime}} K_{\nu}(R) d R .
$$

The Stokes lines emanating from $R_{c}^{\nu \mu}$ are defined by

$$
\operatorname{Re}\left(S_{\nu}\left[R_{c}^{\nu \mu}, R\right]-S_{\mu}\left[R_{c}^{\nu \mu}, R\right]\right)=0 .
$$

There are three such lines, but only one of them crosses the real axis (we take this as an assumption that must be confirmed by the calculations). Let $R_{x}^{\nu \mu}$ be the point where this happens; usually it lies close to $\operatorname{Re}\left(R_{c}^{v \mu}\right)$. It can be easily seen that the lower channel $\nu$ must be locally open at this point, i.e., $K_{\nu}\left(R_{x}^{\nu \mu}\right)$ must be real (that is why only this case has been considered in the above discussion). Moreover, if both channels are locally open at $R_{x}^{\nu \mu}$, then the Stokes line crosses the real axis under the right angle and connects $R_{c}^{\nu \mu}$ with $\left(R_{c}^{\nu \mu}\right)^{*}$; otherwise the angle can be arbitrary, depending on the relation between the absolute values of $K_{\nu}\left(R_{x}^{\nu \mu}\right)$ and $K_{\mu}\left(R_{x}^{\nu \mu}\right)$, and the Stokes lines emanating from $R_{c}^{\nu \mu}$ and $\left(R_{c}^{\nu \mu}\right) *$ do not coincide, see Fig. 9. The Stueckelberg exponent is

$$
\begin{gathered}
\epsilon_{c}^{\nu \mu}=\exp \left(-\Delta_{c}^{\nu \mu}\right), \\
\Delta_{c}^{\nu \mu}=i\left(S_{\nu}\left[R_{c}^{\nu \mu}, R_{x}^{\nu \mu}\right]-S_{\mu}\left[R_{c}^{\nu \mu}, R_{x}^{\nu \mu}\right]\right),
\end{gathered}
$$

where $\Delta_{c}^{\nu \mu}$ is real positive (recall that $\nu<\mu$ ). It has been assumed in Eqs. (61) and (62) that $K_{\nu}\left(R_{c}^{\nu \mu}\right)=K_{\mu}\left(R_{c}^{\nu \mu}\right)$; in the case if $K_{\nu}\left(R_{c}^{\nu \mu}\right)=-K_{\mu}\left(R_{c}^{\nu \mu}\right)$ the sign of the $S_{\mu}$ term should be changed. The Stokes lines emanating from a real turning point $R_{t}^{\nu}$ are defined by

$$
\operatorname{Re}\left(S_{\nu}\left[R_{t}^{\nu}, R\right]\right)=0 .
$$

One of them lies on the real axis to the left (right) of $R_{t}^{\nu}$ in the case of a left-hand (right-hand) turning point, see Fig. 9. The tunneling exponent is

$$
\epsilon_{t}^{\nu \mu}=\exp \left(-\left|\Delta_{t}^{\nu \mu}\right|\right), \quad \Delta_{t}^{\nu \mu}=S_{\mu}\left[R_{t}^{\mu}, R_{x}^{\nu \mu}\right],
$$

where $\Delta_{t}^{\nu \mu}$ is imaginary negative (positive) for a left-hand (right-hand) turning point. Stueckelberg's connection matrix is given by 


$$
N(\epsilon)=\left(\begin{array}{cc}
\sqrt{1-\epsilon^{2}} & \epsilon \\
-\epsilon & \sqrt{1-\epsilon^{2}}
\end{array}\right),
$$

where $\epsilon$ is the amplitude of nonadiabatic transition for a single passage across the Stokes line. The difference in signs of the off-diagonal elements arises from a difference in the behavior of the adiabatic basis functions under the analytic continuation around a crossing point [35], see Eqs. (52). We have neglected the so-called dynamic phase $[2,3]$, which is $O(h)$, as well as higher order corrections [5] to the off diagonal elements because they are beyond the leading order approximation, but retained exponentially small terms in the diagonal elements. Strictly speaking, this is not quite consistent with the leading order approximation, but such an approach preserves unitarity of the scattering matrix.

The strategy of our SC calculations consists of the following. Let $R_{\min }<\cdots<R_{k}<\cdots<R_{\max }$ denote the ordered set of all $R_{t}^{\nu}$ and $R_{x}^{\nu \mu}$ for all active channels. For each $\nu$ $=1, \ldots, N_{\text {act }}$, we construct a global SC solution $\bar{\psi}_{\nu}$ to Eq. (36) satisfying

$$
\left.\bar{\psi}_{\nu}\right|_{R<R_{\min }}=e^{i \pi / 4} \psi_{\nu}^{-}\left(R_{\min }\right),
$$

where the argument of $\psi_{\nu}^{-}$indicates the reference point, i.e., the lower limit of integration in Eq. (44). This is done by propagating a matrix A of coefficients in Eq. (59) from $R_{\text {min }}$ to $R_{\max }$, as described below. Solutions $\bar{\psi}_{\nu}$ are real and satisfy the regularity boundary conditions at $R \rightarrow 0$. Having $N_{\text {act }}$ solutions $\bar{\psi}_{\nu}$, we construct $N_{\mathrm{op}} \leqslant N_{\text {act }}$, their linear combinations $\psi_{\nu}$ satisfying the physical asymptotic boundary conditions

$$
\left.\psi_{\nu}\right|_{R>R_{\max }}=\psi_{\nu}^{-}\left(R_{\max }\right)-\sum_{\mu=1}^{N_{\mathrm{act}}} \bar{S}_{\mu \nu} \psi_{\mu}^{+}\left(R_{\max }\right),
$$

where $N_{\mathrm{op}}$ is the number of open channels. The reduced scattering matrix $\overline{\mathbf{S}}$ differs from $\mathbf{S}$ by phase factors that are immaterial for calculating inelastic transition probabilities, see Eq. (100) below. $\overline{\mathbf{S}}$ is given in terms of $\mathbf{A}$ by

$$
\overline{\mathbf{S}}=i \mathbf{A}(\mathbf{A} *)^{-1} .
$$

The amplitude matrix $\mathbf{A}$ is calculated using the following procedure.

(1) At the initial point $R=R_{\min }$, set $\mathbf{A}$ equal to a unit matrix of the dimension $N_{\text {act }}$.

(2) Propagation from $R_{k}$ to $R_{k+1}$ :

$$
\mathbf{A}\left(R_{k+1}\right)=\mathbf{E}\left(R_{k}, R_{k+1}\right) \mathbf{A}\left(R_{k}\right),
$$

where $\mathbf{E}\left(R_{k}, R_{k+1}\right)$ is a diagonal matrix with the elements $\exp \left(i S_{\nu}\left[R_{k}, R_{k+1}\right]\right)$ and 1 for channels that are locally open and closed in the interval $R_{k}<R<R_{k+1}$, respectively. This corresponds to changing the reference point for locally open channels.

(3) Nonadiabatic transition at $R_{x}^{\nu \mu}$ if the upper channel $\mu$ is locally open:

$$
\mathbf{A}\left(R_{x}^{\nu \mu}+0\right)=\mathbf{N}_{\nu \mu}(\boldsymbol{\epsilon}) \mathbf{A}\left(R_{x}^{\nu \mu}-0\right),
$$

where $\mathbf{N}_{\nu \mu}(\epsilon)$ is a matrix that acts as $N(\boldsymbol{\epsilon})$ on channels $\nu$ and $\mu$ and as a unit matrix on all the other channels, and $\epsilon=\epsilon_{c}^{\nu \mu}$.
(4) Nonadiabatic transition at $R_{x}^{\nu \mu}$ if the upper channel $\mu$ is locally closed and $R_{x}^{\nu \mu}<R_{t}^{\mu}$ (left-hand turning point): apply Eq. (70) with $\epsilon=\epsilon_{c}^{\nu \mu} \epsilon_{t}^{\nu \mu}$.

(5) Nonadiabatic transition at $R_{x}^{\nu \mu}$ if the upper channel $\mu$ is locally closed and $R_{t}^{\mu}<R_{x}^{\nu \mu}$ (right-hand turning point): to each column $\mu^{\prime} \neq \mu$ of $\mathbf{A}$ add column $\mu$ multiplied by $\epsilon \operatorname{Re}\left(A_{\nu \mu^{\prime}}\right) / \operatorname{Re}\left(A_{\mu \mu}\right)$ and then apply Eq. (70), where $\epsilon$ $=\epsilon_{c}^{\nu \mu} \epsilon_{t}^{\nu \mu}$.

(6) Closing a channel $\nu$ at the right-hand turning point $R_{t}^{\nu}$ : from each column $\nu^{\prime} \neq \nu$ of $\mathbf{A}$ subtract column $\nu$ multiplied by $\operatorname{Re}\left(A_{\nu \nu^{\prime}}\right) / \operatorname{Re}\left(A_{\nu \nu}\right)$.

(7) At the final point $R=R_{\max }$, delete all rows and columns of $\mathbf{A}$ that correspond to closed channels. The result is a square matrix of the dimension $N_{\mathrm{op}}$. It should be substituted into Eq. (68) to obtain the reduced scattering matrix.

To implement this procedure, one needs to find the Stokes lines (61). This can be easily done using the procedure of numerical analytic continuation described in Sec. IV C 1. The connection (70) could be applied, e.g., at $\operatorname{Re}\left(R_{c}^{\nu \mu}\right)$ instead of $R_{x}^{\nu \mu}$, which would make finding the Stokes lines not necessary. However, the calculations are very easy, so we prefer to act as described above to keep the whole situation including the positions of Stokes lines under control. It can be easily seen that $\mathbf{N}_{\nu \mu}^{-1}(\epsilon)=\mathbf{N}_{\nu \mu}^{T}(\epsilon)$ and $\mathbf{E}^{-1}\left(R_{k}, R_{k+1}\right)$ $=\mathbf{E}^{*}\left(R_{k}, R_{k+1}\right)$, which guarantees that $\overline{\mathbf{S}}$ obtained from Eq. (68) is unitary.

\section{E. Analysis of hidden crossings}

From the mathematical viewpoint, the results of Landau [20], who showed that in the SC approximation inelastic transitions occur via branch points of the adiabatic potentials, and Stueckelberg [21], who solved the problem of connection of the SC solutions across the Stokes lines emanating from such a branch point, are self-sufficient in the sense that they enable one to calculate transition probabilities. However, in physics one tries to look deeper and asks what is the cause of the appearance of branch points, when and where one should expect them to appear, and eventually what are the physical mechanisms of the transitions. These questions were first raised in the context of the theory of slow ion-atom collisions by Solov'ev, which has led him to the discovery of hidden crossings [22]. In this section we show that in our problem the branch points in case A are hidden crossings of the well-known $T$ series, and those in case B present a generalization that will be called the complex $T$ series.

Before proceeding, let us comment on the terminology used in this field, which is rather confusing. The "branch point" is a clear mathematical term, but it lacks physical contents. In the physical literature on nonadiabatic transitions one can often meet a more vague term "crossing point" that emphasizes the fact that two adiabatic potentials cross, i.e., have the same value at some point. However, it is wellknown that usually they cannot cross on the real axis. This circumstance is taken into account by the probably most frequently used term "avoided crossing"; in many papers and books the very concept of nonadiabatic transition is illustrated by the Landau-Zener type avoided crossing of potential curves. The term "hidden crossing" was originally used 
in the narrow sense of the word to distinguish branch points that lie deeper in the complex plane and do not reveal themselves as typical avoided crossings on the real axis. However, later on it was realized from studies of the two-center Coulomb problem [22,43-49] that there is an essential difference between hidden crossings and isolated branch points, namely, hidden crossings appear in well-organized series with a common mechanism, and this term has acquired a broader sense designating the physical phenomenon itself responsible for their appearance. The purpose of this section is to clarify the mechanism of nonadiabatic transitions in our problem by showing that crossing points discussed above are hidden crossings in the latter sense of the word.

To analyze the origin of branch points of the eigenvalue $U(R)$ of Eqs. (47a) and (47b) in the situation when the analytic solution is not available there is no better tool than the asymptotic theory. Let us rewrite Eq. (47a) as

$$
\left[\frac{d^{2}}{d \phi^{2}}+\lambda F(\phi)\right] \Phi(\phi)=0
$$

where

$$
F(\phi)=2[U-R C(\phi)] .
$$

We have introduced here a formal asymptotic parameter $\lambda$ $=1$ and are going to discuss the asymptotic solutions of Eq. (71) for $\lambda \rightarrow \infty$; keeping this in mind, we omit $\lambda$ from the equations below. We shall treat $R$ and $U$ as independent complex parameters until a quantization condition defining the function $U(R)$ is obtained. The structure of the asymptotic solution is determined by the analytical properties of $F(\phi)$ as a function of complex $\phi$ in the region near the interval 0 $\leqslant \phi \leqslant \pi / 2$. It can be seen from Eqs. (27) that $F(\phi)$ has two simple zeros at $\phi_{1}$ and $\phi_{2}$ and two simple poles at $\phi_{3}=0$ and $\phi_{4}=\pi / 2$, see Fig. 10; thus we deal with a four transition point problem. Besides, function $F(\phi)$ has an extremum at $\phi_{0}$, where $C^{\prime}\left(\phi_{0}\right)=0$. The poles $\phi_{3}$ and $\phi_{4}$ have the same positions for all systems; the extremum $\phi_{0}$ is a property of the effective charge $C(\phi)$, so its position depends only on the system; the zeros $\phi_{1}$ and $\phi_{2}$ depend in addition on the parameters $R$ and $U$, so they are movable. Hidden crossings of the type we are going to discuss are characterized by the condition

$$
U \approx R C\left(\phi_{0}\right),
$$

i.e., the eigenvalue $U$ must be close to the value of the potential energy $R C(\phi)$ at its extremum. The validity of this condition is illustrated for crossing points of the main series in systems with $h=2 / 3$ in Table II; the situation for other values of $h$ is similar. In case A, the extremum of $C(\phi)$ lies on the real axis at the point $\phi_{0}$ where the potential barrier separating two Coulomb wells reaches its maximum, see Fig. 2 . This corresponds to the well-known situation giving rise to the $T$ (the name comes from the top of the potential barrier) series of hidden crossings [6]. In case $B$, there is no potential barrier on the real axis, see Fig. 2, but $C(\phi)$ still has an extremum, now at a complex point $\phi_{0}$. Although this situation is formally similar to that in case A, it introduces new features and, as we shall see below, leads to a qualitatively
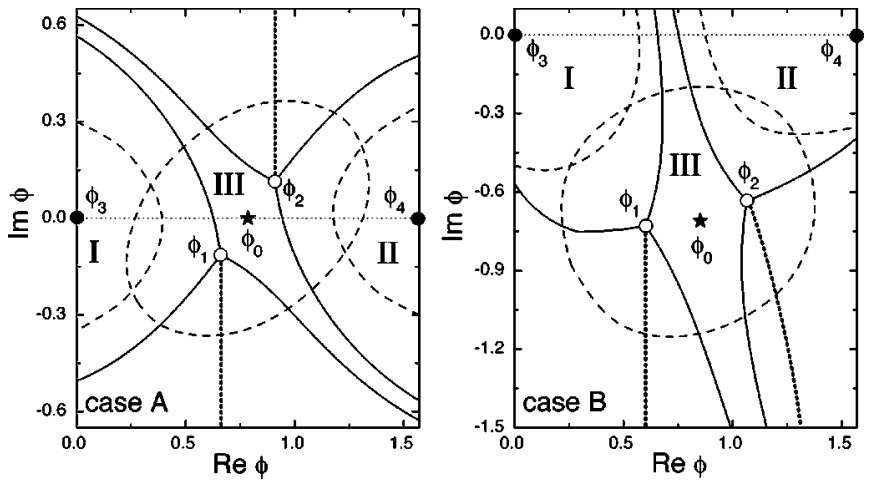

FIG. 10. The analytical structure of function (72) in complex $\phi$ plane. Stars, the extremum; open circles, simple zeros; closed circles, simple poles; solid lines, Stokes lines defined by Eq. (76); dotted lines, branch cuts; and dashed lines, approximate boundaries of the regions where different comparison equations were used. The figures show actual positions of the Stokes lines for $h=2 / 3$ and the values of $R$ and $U$ corresponding to the tenth hidden crossing from the main series, see Table I; this situation is typical.

different distribution of crossing points in the complex $R$ plane; hidden crossings in this case will be called the complex $T$ series. In fact, in case B function $C(\phi)$ has another extremum near the interval $0 \leqslant \phi \leqslant \pi / 2$ at the complex conjugate point $\phi_{0}^{*}$. As can be seen from Eq. (73), this extremum is associated with hidden crossings having complex conjugate values of $R$ and $U$, so it is sufficient to consider only the one at $\phi_{0}$.

Condition (73) means that zeros $\phi_{1}$ and $\phi_{2}$ lie close to the extremum $\phi_{0}$, and hence to each other. Thus we need an asymptotic solution of Eq. (71) that is uniform in the distance between $\phi_{1}$ and $\phi_{2}$. Such a solution can be constructed using the comparison equation method; see, e.g., [50-52]. Let us introduce the action for the motion in $\phi$,

$$
s\left[\phi^{\prime}, \phi^{\prime \prime}\right]=\int_{\phi^{\prime}}^{\phi^{\prime \prime}} \sqrt{F(\phi)} d \phi
$$

and actions between pairs of transition points $\phi_{i}, i=1, \ldots, 4$,

TABLE II. The validity of Eq. (73) for hidden crossings of the main series in systems with $h=2 / 3$. The values of $R_{c}^{\nu, \nu+1}$ and $U_{c}^{\nu, \nu+1}$ correspond to the QM results from Table I.

\begin{tabular}{cccc}
\hline \hline & \multicolumn{3}{c}{$U_{c}^{\nu, \nu+1} /\left[R_{c}^{\nu, \nu+1} C\left(\phi_{0}\right)\right]$} \\
\cline { 2 - 4 }$\nu$ & Case A, $\sigma=+$ & Case A, $\sigma=-$ & Case B \\
\hline 1 & $(1.084,0.616)$ & $(1.198,1.240)$ & $(1.602,0.329)$ \\
2 & $(1.032,0.305)$ & $(1.092,0.643)$ & $(1.286,0.174)$ \\
3 & $(1.017,0.201)$ & $(1.055,0.437)$ & $(1.185,0.119)$ \\
4 & $(1.011,0.149)$ & $(1.037,0.331)$ & $(1.135,0.090)$ \\
5 & $(1.008,0.118)$ & $(1.027,0.267)$ & $(1.106,0.073)$ \\
10 & $(1.002,0.057)$ & $(1.009,0.135)$ & $(1.050,0.037)$ \\
\hline \hline
\end{tabular}




$$
I_{i j}=s\left[\phi_{i}, \phi_{j}\right] .
$$

The branch of the square root function in Eq. (74) is determined by the condition that it takes real positive values for real $R>0, U$, and $\phi \rightarrow+0$, and by the cuts shown in Fig. 10 . Note that only three of six quantities $I_{i j}$ are independent, e.g., $I_{34}=I_{31}+I_{12}+I_{24}$; in the symmetric case $I_{31}=I_{24}$, and only two of them remain independent. The structure of the Stokes lines emanating from the zeros $\phi_{1}$ and $\phi_{2}$ and defined by

$$
\operatorname{Re}\left(s\left[\phi_{1,2}, \phi\right]\right)=0
$$

is illustrated in Fig. 10. The asymptotic solutions in regions I and II near the poles satisfying boundary conditions (47b) can be expressed in terms of the Bessel function $J_{1}(z)$,

$$
\Phi_{\mathrm{I}, \mathrm{II}}(\phi)=\left(\frac{s_{\mathrm{I}, \mathrm{II}}^{2}}{F(\phi)}\right)^{1 / 4} J_{1}\left(s_{\mathrm{I}, \mathrm{II}}\right),
$$

where the local variables $s_{\mathrm{I}}$ and $s_{\mathrm{II}}$ are

$$
s_{\mathrm{I}}=s\left[\phi_{3}, \phi\right], \quad s_{\mathrm{II}}=s\left[\phi, \phi_{4}\right] .
$$

An asymptotic solution in region III including two zeros and the extremum of $F(\phi)$ can be expressed in terms of Kummer's function $M(a, b, z)$,

$$
\Phi_{\mathrm{III}}(\phi)=\left(\frac{s_{\mathrm{III}}^{2}+a}{F(\phi)}\right)^{1 / 4}\left[A_{+} y_{+}\left(s_{\mathrm{III}}\right)+A_{-} y_{-}\left(s_{\mathrm{III}}\right)\right],
$$

where $A_{ \pm}$are arbitrary constants, functions $y_{ \pm}(s)$ are defined by

$$
y_{ \pm}(s)=s^{(1 \mp 1) / 2} \exp \left(-\frac{i s^{2}}{2}\right) M\left[\frac{2 \mp 1+i a}{4}, \frac{2 \mp 1}{2}, i s^{2}\right]
$$

and satisfy $y_{ \pm}(-s)= \pm y_{ \pm}(s)$, the local variable $s_{\mathrm{III}}$ is defined implicitly by

$$
\int_{-i \sqrt{a}}^{s_{\text {III }}} \sqrt{s^{2}+a} d s=s\left[\phi_{1}, \phi\right],
$$

and $a$ is the key parameter characterizing the distance between the zeros $\phi_{1}$ and $\phi_{2}$,

$$
a=-\frac{2 i}{\pi} I_{12} .
$$

The global solution can be now obtained by matching the above local solutions in the overlapping parts of the regions I, II, and III. We note that different asymptotics of Kummer's function must be used for matching the solutions $\Phi_{\mathrm{II}}(\phi)$ and $\Phi_{\text {III }}(\phi)$ in cases A and B because of the difference in the position of the corresponding matching region with respect to the Stokes lines, see Fig. 10. Applying matching we find that the global solution exists only if $R$ and $U$ satisfy certain quantization condition. In case $\mathrm{A}$, this condition reads

$$
\cos \left[I_{31}+I_{24}-\varphi(a)\right]=\frac{\cos \left(I_{31}-I_{24}\right)}{\sqrt{1+\exp (\pi a)}},
$$

which in the symmetric case for the permutation symmetry $\sigma= \pm$ reduces to

$$
\sin \left[I_{31}-\varphi_{\sigma}(a)+\frac{\sigma \pi}{8}\right]=0,
$$

and in case B we obtain

$$
e^{2 i I_{31}}+e^{-2 i I_{14}}=e^{i \varphi(a)} \sqrt{1+\exp (\pi a)},
$$

where $\varphi_{\sigma}(a)$ and $\varphi(a)$ are dynamic phases for the parabolic barrier,

$$
\begin{aligned}
\varphi_{\sigma}(a)=\frac{i}{2} \ln \left(\frac{\Gamma((2-\sigma-i a) / 4)}{\Gamma((2-\sigma+i a) / 4)}\right)-\frac{a}{4}\left(\ln \frac{a}{4}-1+i \pi\right), \\
\varphi(a)=\varphi_{+}(a)+\varphi_{-}(a)=\frac{i}{2} \ln \left(\frac{\Gamma((1-i a) / 2)}{\Gamma((1+i a) / 2)}\right) \\
-\frac{a}{2}\left(\ln \frac{a}{2}-1+i \pi\right) .
\end{aligned}
$$

Equations (80)-(82) present uniform semiclassical (USC) quantization conditions defining the eigenvalues of Eq. (71) for any value of $a$. Let us show that for real $R$ and $U$ in the limit $|a| \rightarrow \infty$ they turn into the usual (leading order) BohrSommerfeld (BS) quantization conditions on the real axis. Indeed, the dynamic phases for $|a| \rightarrow \infty$ behave as

$$
=\left\{\begin{array}{c}
\frac{\sigma \pi}{8}+\frac{1}{24 a}+O\left(a^{-2}\right),-\frac{3 \pi}{2}<\arg a<-\frac{\pi}{2}, \\
-\frac{i \pi a}{4}-\frac{\sigma \pi}{8}+\frac{1}{24 a}+O\left(a^{-2}\right),-\frac{\pi}{2}<\arg a<\frac{\pi}{2} .
\end{array}\right.
$$

In case A, $a$ is real and its sign depends on whether $U$ lies below or above the maximum of the potential barrier. For $U<R C\left(\phi_{0}\right)$ we have $a \rightarrow-\infty$, and Eq. (80) reduces to

$$
\sin \left(I_{31}\right) \sin \left(I_{24}\right)=0 .
$$

This yields the BS quantization conditions separately for each of the two potential wells (it should be taken into account that there is a simple pole on one end of the intervals of quantization $0<\phi<\phi_{1}$ and $\left.\phi_{2}<\phi<\pi / 2\right)$. For $U$ $>R C\left(\phi_{0}\right)$ we have $a \rightarrow+\infty$, and Eq. (80) takes the form

$$
\cos \left(I_{34}\right)=0 \text {. }
$$

This coincides with the BS quantization condition for the whole interval $0<\phi<\pi / 2$ between the two poles. In case B, the parameter $a$ is complex with a negative real part, $I_{14}$ is imaginary negative, and we obtain from Eq. (82)

$$
e^{2 i I_{31}}=1 .
$$

This coincides with the BS quantization condition for the interval $0<\phi<\phi_{1}$ in the single potential well available in this case.

The quantization conditions (80)-(82) can be presented in the form 


$$
Q(R, U)=0,
$$

which defines a multivalued analytic function $U(R)$. The branch points of $U(R)$ simultaneously satisfy Eq. (88a) and

$$
\frac{\partial Q(R, U)}{\partial U}=0
$$

In general, it is a rather difficult computational task to find the solutions to these equations because of the multivaluedness of the dynamic phases (83); the only known to us calculation of this type was reported in [53]. However, in our case a very good initial guess is available which is provided by the QM results, so the root finding procedure converges in a few iterations. The results of our calculations for the main series of hidden crossings in systems with $h=2 / 3$ are presented in Table I. The agreement between the QM and USC results is impressive; it becomes better for higher members of the series, but is not bad even for the lowest one. The agreement for secondary series and other values of $h$ is similar.

Having confirmed that the USC quantization conditions (80)-(82) nicely reproduce the positions of hidden crossings, we can use them to investigate the dependence of $R_{c}^{\nu \mu}$ and $U_{c}^{\nu \mu}$ on the quantum numbers $\nu<\mu$. To this end, let us discuss the asymptotic solutions of Eqs. (88a) and (88b) for $\nu$ $\rightarrow \infty$. It is convenient to introduce instead of $R, U$, and $F(\phi)$ new parameters

$$
\rho=\sqrt{2 R C_{2}}, \quad \varepsilon=\frac{U-R C_{0}}{R C_{2}},
$$

and a new function

$$
f(\phi)=\sqrt{\frac{C_{0}-C(\phi)}{C_{2}}}=\left(\phi_{0}-\phi\right)\left[1+O\left(\phi_{0}-\phi\right)\right],
$$

where $C_{0}=C\left(\phi_{0}\right)$ and $C_{2}=-\frac{1}{2} C^{\prime \prime}\left(\phi_{0}\right)$. Then Eq. (72) takes the form

$$
F(\phi)=\rho^{2}\left[\varepsilon+f^{2}(\phi)\right] .
$$

The solutions of Eqs. (88a) and (88b), i.e., the hidden crossings, can be labeled by two integers, $\nu=1,2,3, \ldots$ and $n$ $=0,1,2, \ldots$, that enumerate, respectively, columns and rows in Figs. 3 and 4. We are interested in the solutions satisfying

$$
\nu \rightarrow \infty, \quad n=O\left(\nu^{0}\right), \quad \rho=O(\nu), \quad \varepsilon=O\left(\nu^{-1}\right) .
$$

They can be obtained using the expansions (we consider only the symmetric case in case A, so it is assumed that $I_{31}=I_{24}$ )

$$
\begin{gathered}
I_{31}=\rho\left[p_{0}+p_{1} \varepsilon \ln \varepsilon+p_{2} \varepsilon+O\left(\varepsilon^{2} \ln \varepsilon\right)\right], \\
a=\rho \varepsilon[1+O(\varepsilon)],
\end{gathered}
$$

where

$$
p_{0}=\int_{0}^{\phi_{0}} f(\phi) d \phi, \quad p_{1}=-\frac{1}{4}
$$

$$
p_{2}=\frac{1}{2} \int_{0}^{\phi_{0}}\left(\frac{1}{f(\phi)}-\frac{1}{\phi_{0}-\phi}\right) d \phi+\frac{1}{2} \ln 2 \phi_{0}+\frac{1-i \pi}{4} .
$$

Omitting the details (an example of the derivation can be found in [54]), the result reads

$$
\begin{aligned}
\rho_{\nu n}= & \frac{\pi}{p_{0}} \nu-\frac{a_{n}}{4 p_{0}} \ln \nu+\frac{i}{2 p_{0}} \ln \ln \nu \\
+ & \frac{1}{2 p_{0}}\left(\frac{a_{n}}{2} \ln \frac{a_{n} p_{0}}{\pi}-2 a_{n} p_{2}+\frac{\pi}{2}+i b_{n}\right)+O\left(\frac{1}{\ln \nu}\right), \\
\varepsilon_{\nu n} & =\frac{a_{n} p_{0}}{\pi} \frac{1}{\nu}-\frac{2 i p_{0}}{\pi} \frac{1}{\nu \ln \nu}+O\left(\frac{1}{\nu \ln ^{2} \nu}\right)
\end{aligned}
$$

where in case A for the permutation symmetry $\sigma$

$$
\begin{gathered}
a_{n}=-i(2-\sigma+4 n), \\
b_{n}=1-\ln [n ! \Gamma(1-\sigma / 2+n) / 2] \\
+(1-\sigma / 2+2 n)[\ln (1 / 2-\sigma / 4+n)-1],
\end{gathered}
$$

and in case B

$$
\begin{gathered}
a_{n}=-i(1+2 n), \\
b_{n}=1-\ln [\sqrt{2 \pi} n !]+(1 / 2+n)[\ln (1 / 2+n)-1] .
\end{gathered}
$$

From Eqs. (93) and (95) we obtain

$$
a=a_{n}-\frac{2 i}{\ln \nu}+O\left(\frac{1}{\ln ^{2} \nu}\right),
$$

so $a$ approaches $a_{n}$ as $\nu$ grows. For $a=a_{n}$ the argument of the upper gamma function in Eqs. (83) is equal to a nonpositive integer $-n$, thus hidden crossings described by Eqs. (95) lie near its poles. Each pole gives rise to a series of hidden crossings converging to it as $\nu$ grows, with $n$ numerating the poles and $\nu$ numerating the members of the series. This explains the meaning of the quantum numbers $\nu$ and $n$. The results of our calculations using Eqs. (89) and (95) are presented in Table I. The agreement between the asymptotic (AS) and QM results is worse than in the case of the USC results, but it becomes better as $\nu$ grows, slowly because the error terms in Eqs. (95) decay slowly. In the extreme limit $\nu \rightarrow \infty$, the positions of the hidden crossings of the main series $(n=0)$ in case A are given by

$$
R_{c}^{\nu, \nu+1}=\frac{\pi^{2}}{2 C_{2} p_{0}^{2}}\left(\nu^{2}+i \frac{2-\sigma}{2 \pi} \nu \ln \nu\right)+O(\nu \ln \ln \nu),
$$

and in case B by

$$
R_{c}^{\nu, \nu+1}=\frac{\pi^{2} \nu^{2}}{2 C_{2} p_{0}^{2}}+O(\nu \ln \nu) .
$$

The difference of these formulas is explained by the fact that in case A the coefficients $C_{2}$ and $p_{0}$ are real, so two terms in Eq. (99a) are needed to obtain both real and imaginary parts 
of $R_{c}^{\nu, \nu+1}$, while in case B they are complex, so it is sufficient to leave only one term in Eq. (99b). This leads to an essential difference in the distribution of hidden crossings in the two cases, see Figs. 3 and 4. A relation between the real and imaginary parts of $R_{c}^{\nu, \nu+1}$ dictated by Eq. (99a) is typical for the $T$ series of hidden crossings [6,52]; that following from Eq. (99b) characterizes the complex $T$ series.

\section{SCATTERING CALCULATIONS}

This section presents the results of scattering calculations. The QM results reported below were obtained using the CTBC program [19], and the SC results were obtained using the procedure described in Sec. IV D. Inelastic processes (13) will be characterized by their probabilities

$$
p_{\nu \mu}=\left|S_{\nu \mu}\right|^{2},
$$

where the symmetrized scattering matrix (16) is used in case A. We shall consider $p_{\nu \mu}$ as functions of the effective quantum number

$$
n(E)=(-2 E)^{-1 / 2} \text {. }
$$

As can be seen from Eqs. (15) and (28), the energy intervals between thresholds of consecutive channels $E_{\nu} \leqslant E \leqslant E_{\nu+1}$ correspond to $\nu \leqslant n(E) \leqslant \nu+1$, so $n(E)$ provides a more convenient energy scale below the three-body disintegration threshold $E=0$. The following results illustrate the conclusions made on the basis of more extensive calculations for different systems and processes in the energy range up to $n(E)=6$.

The first feature to be observed is the dominant role of the main series of hidden crossings in the SC calculations. The same transition can occur via several different paths. For example, the transition between states 1 and 3 in case A can proceed in one step, via the point $R_{c}^{13}$, or in two steps, via $R_{c}^{12}$ and $R_{c}^{23}$, see Fig. 3 ; in case B it can proceed in two steps via $R_{c}^{12}$ and one of the two points $R_{c}^{23}$, see Fig. 4. Each crossing point $R_{c}^{\nu \mu}$ is characterized by the amplitude $\epsilon$ of the nonadiabatic transition in Eq. (65), which is equal to Stueckelberg's exponent (62), if the upper channel $\mu$ is locally open at $R_{x}^{v \mu}$, or to a product of that and the tunneling exponent (64), if it is locally closed. The amplitude for a multistep path apart from a phase factor is equal to the product of amplitudes for each step. Our calculations show that the maximum amplitude always corresponds to the path going via crossing points only of the main series, which can be confirmed by the asymptotic analysis of Sec. IV E. Strictly speaking, only the maximum amplitude path for each transition should be taken into account in the leading order approximation, which means that the SC results are subject to an error arising from the effect of crossing points of secondary series. This effect is exponentially small compared to that of the main series. However, now we are not satisfied with such an asymptotic estimate of the error and wish to know its actual numerical magnitude. Our calculations show that the effect of secondary series is always negligible, it cannot be even seen in the scale of a typical figure. So in the calculations below only hidden crossings of the main series are included, in accord with the prescription of the leading order approximation.

Our main goal is to demonstrate how the SC and QM results converge as the asymptotic parameter $h$ tends to zero. To this end, we consider the transition between states 1 and 2 in the energy interval $2 \leqslant n(E) \leqslant 3$, where only this inelastic transition is possible, and compare the SC and QM results for $p_{12}$ for several systems with decreasing values of $h$. The results for case A, $\sigma=+$, are shown in Fig. 11. For $h=1$, which corresponds to the least favorable situation for the SC approximation, the agreement between the SC and QM results is rather qualitative than quantitative. The energy dependence of $p_{12}$ in this case is dominated by resonances. In the SC calculations, resonances result from the interaction with closed active channels (channel 3 in the present case) in the energy interval $V_{\nu}^{\min }<E<E_{\nu}$. Although the SC results fairly well reproduce the shape of resonances, their positions are essentially shifted. On average, the SC results overestimate the QM results by about a factor of 2 . For $h=1 / 2$, the agreement becomes much better. Resonances, whose widths exponentially decay with $h$, still play an important role, but do not dominate the behavior of $p_{12}$ anymore. The shift between their positions in the $\mathrm{SC}$ and QM results decreases as $O\left(h^{2}\right)$, so it became smaller. On average, the difference between the SC and QM results is less than 20\%, but now the former are lower. For $h=1 / 4$, resonances are very narrow and are not resolved in the figure. The agreement is almost perfect, the difference is only $3 \%$. This seems to demonstrate the expected convergence. However, its nonmonotonic character prompts us to consider smaller variations of $h$. When $h$ is decreased further by a small amount to the value $h$ $\approx 0.23$, which corresponds to the mass-ratio $M=14$, the SC and QM results diverge violently. An explanation for such a behavior lies in Stueckelberg's oscillations. To illustrate this, let us consider $p_{12}$ as a function of $h$ for a fixed value of $E$. We chose the point $n(E)=2.3$ that lies below the region of resonances. Only two channels ( 1 and 2$)$ are active at this energy, only one crossing point $\left(R_{c}^{12}\right)$ is operative, and for all values of $h$ channel 2 is locally open at $R_{x}^{12}$. In this case, the procedure described in Sec. IV D yields the original Stueckelberg's result [21] for the transition probability

$$
p_{12}=4 p(1-p) \sin ^{2} \delta,
$$

where

$$
p=\exp \left(-2 \Delta_{c}^{12}\right), \quad \delta=S_{1}\left[R_{t}^{1}, R_{x}^{12}\right]-S_{2}\left[R_{t}^{2}, R_{x}^{12}\right] .
$$

The oscillating factor $\sin ^{2} \delta$ in Eq. (102) describes the interference of two paths of the nonadiabatic transition that can occur on the way in (decreasing $R$ ), or out (increasing $R$ ). The parameters $\Delta_{c}^{12}$ and $\delta$ depend on $h$. The main dependence comes from the factor $h^{-1}$ in Eq. (60), but there is also a weak dependence caused by the dependence on $h$ of the effective charge. Taking into account Eqs. (27), they can be expanded as

$$
\Delta_{c}^{12}=\frac{\Delta_{0}}{h}+O(h), \quad \delta=\frac{\delta_{0}}{h}+O(h),
$$

where the coefficients $\Delta_{0}$ and $\delta_{0}$ for the given energy can be calculated numerically. Figure 12 illustrates how the SC and 


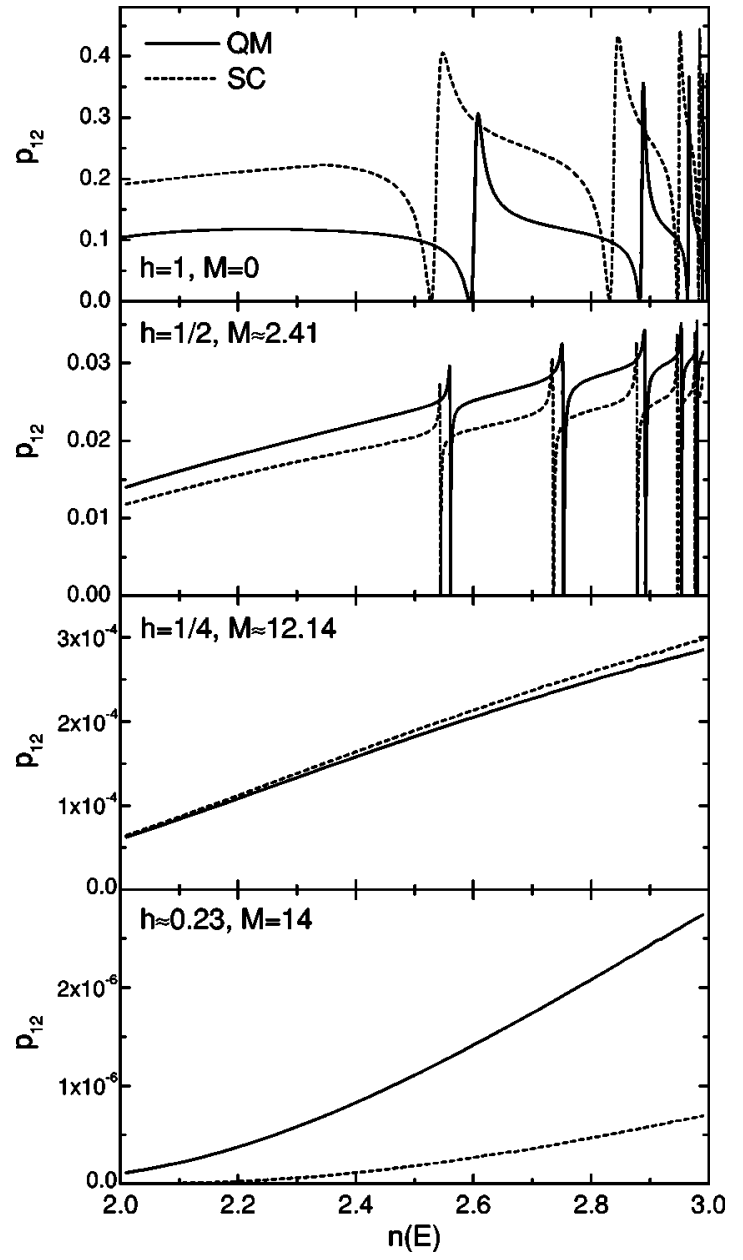

FIG. 11. The probability (100) of transition between states 1 and 2 as a function of the effective quantum number (101) for several values of $h$ in case A, $\sigma=+$. The asymptotic parameter $h$ and the mass ratio $M$ are related by Eqs. (26). For $h=1 / 4$ and 0.23 resonances are too narrow and are not resolved in the figure.

QM results converge. In the interval of $h$ shown, the value of $p$ in Eq. (102) decreases by four orders of magnitude. To eliminate this strong exponential dependence, we divided the SC and QM results for $p_{12}$ by $4 p(1-p)$, where $p$ was calculated using Eqs. (103) and (104). In the limit $h \rightarrow 0$ the ratio should coincide with $\sin ^{2} \delta$, where $\delta$ is given by Eq. (104). As can be seen from the figure, this function indeed nicely reproduces the oscillatory behavior of the results, and the agreement becomes better as $1 / h$ grows. The agreement between the SC and QM results is generally very good, but there is a small $O(h)$ phase shift in their oscillations, which is consistent with the leading order approximation. As a consequence, the absolute error of the SC results oscillates with the same period and the amplitude decaying as $O(h)$, see the upper panel in Fig. 12. The relative error strongly depends on the phase of the oscillations, and there are unfavorable intervals of $h$ near the minima of $\sin ^{2} \delta$, which corresponds to a destructive interference, where the relative error may become very large even for very small values of $h$. These results add a new dimension to the situation shown in Fig. 11. Now it becomes clear that the nonmonotonic character of conver-

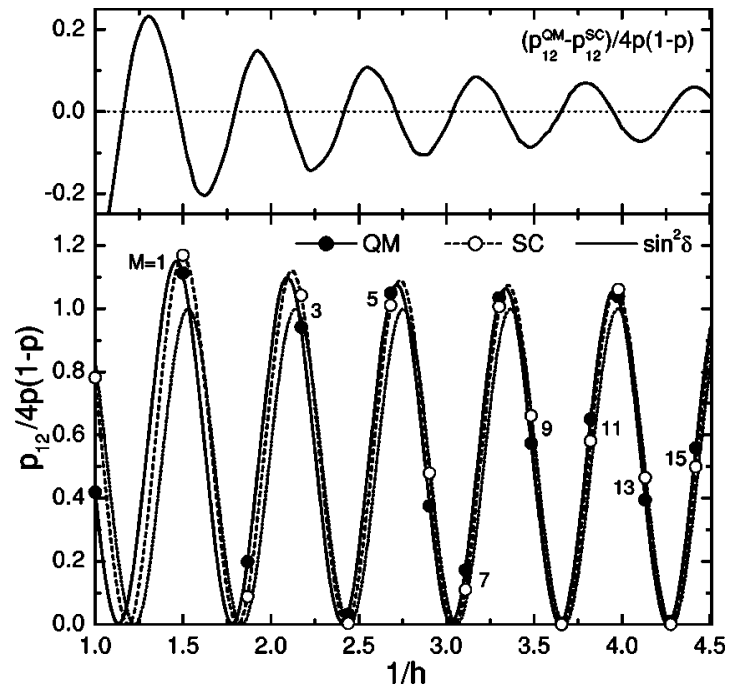

FIG. 12. The probability $p_{12}$ as a function of $1 / h$ for a fixed value of $n(E)=2.3$ in case $\mathrm{A}, \sigma=+$. The $\mathrm{SC}$ and $\mathrm{QM}$ results are divided by $4 p(1-p)$ and compared with $\sin ^{2} \delta$, see Eq. (102), where $p$ and $\delta$ were calculated using Eqs. (104) with $\Delta_{0} \approx 1.288$ and $\delta_{0}$ $\approx 5.136$. The circles show the results for integer values of the mass ratio $M=0,1, \ldots, 15$.

gence of the $\mathrm{SC}$ and $\mathrm{QM}$ results is a manifestation of Stueckelberg's oscillations, and a big difference between them for $h \approx 0.23$ is explained by the fact that this value of $h$ happened to lie very close to a minimum of the oscillating factor in Eq. (102). The results of similar calculations for case A, $\sigma=-$, and case B are shown in Figs. 13 and 14 . Again it can be seen that the agreement between the SC and QM results depends stronger on the phase of the oscillations in $h$ rather than on the value of $h$ itself. Indeed, the difference can be small even for not very small values of the asymptotic parameter, e.g., it is less than $15 \%$ in both cases for $h=2 / 3$, but it becomes large in the unfavorable intervals of $h$ where destructive interference occurs, as in case B for $h=1$ and $1 / 2$. Thus Stueckelberg's oscillations play a crucial role in the understanding of convergence of the SC and QM results.

Let us now demonstrate the agreement between the SC and QM results in a wider energy interval and for other processes. The results of calculations for systems with an intermediate value of the asymptotic parameter $h=2 / 3$ in each of the three cases are shown in Figs. 15-17. In case A, $\sigma=+$, we present the results only up to $n(E)=5$; because of the resonances, at higher energies it becomes difficult to distinguish different processes if they are plotted in the same figure. The results in case A, $\sigma=-$, and case B continue those for $p_{12}$ shown in the middle panels of Figs. 13 and 14. The overall agreement can be characterized as very good, especially taking into account that probabilities of the different processes differ by many orders of magnitude. The SC results nicely reproduce this difference, as well as the shape of the energy dependence of the probabilities. We note that the rapid (exponential) growth of the probabilities just above the thresholds in case B comes from the energy dependence of tunneling exponents (64) and reflects their dependence on the position of turning points $R_{t}^{\mu}$. The SC calculations do well even in cases when the probabilities are too small (less than 


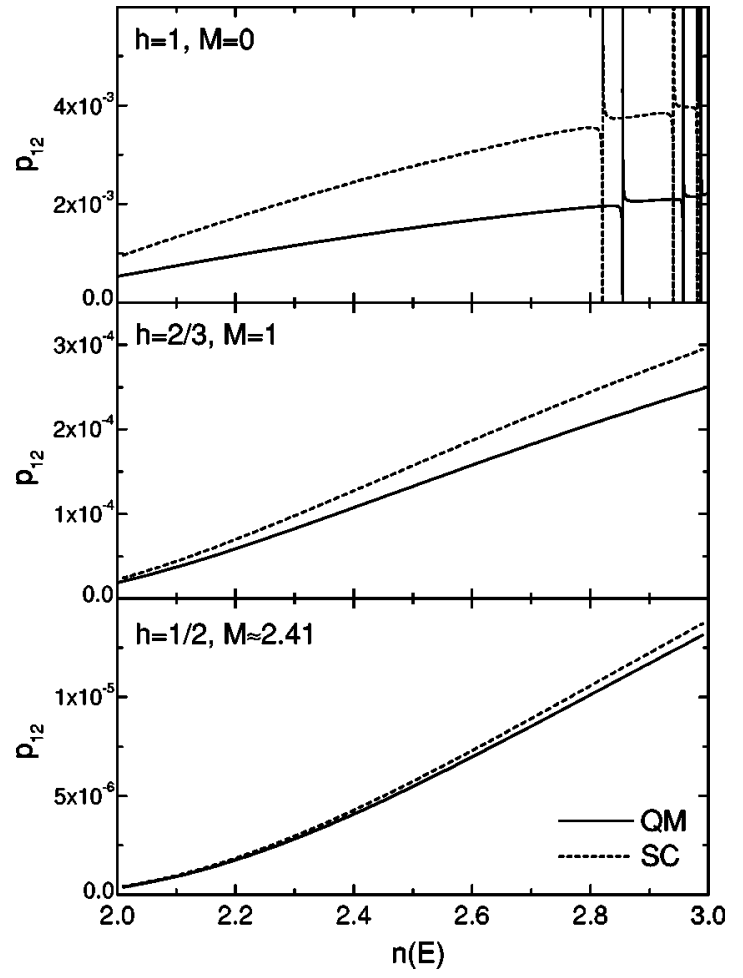

FIG. 13. Same as in Fig. 11, but in case A, $\sigma=-$. For $h=2 / 3$ and $1 / 2$ resonances are not resolved.

$10^{-10}$ ) and our double precision QM calculations fail to converge because of the roundoff errors, see Fig. 17. This situation is typical for other values of $h$.

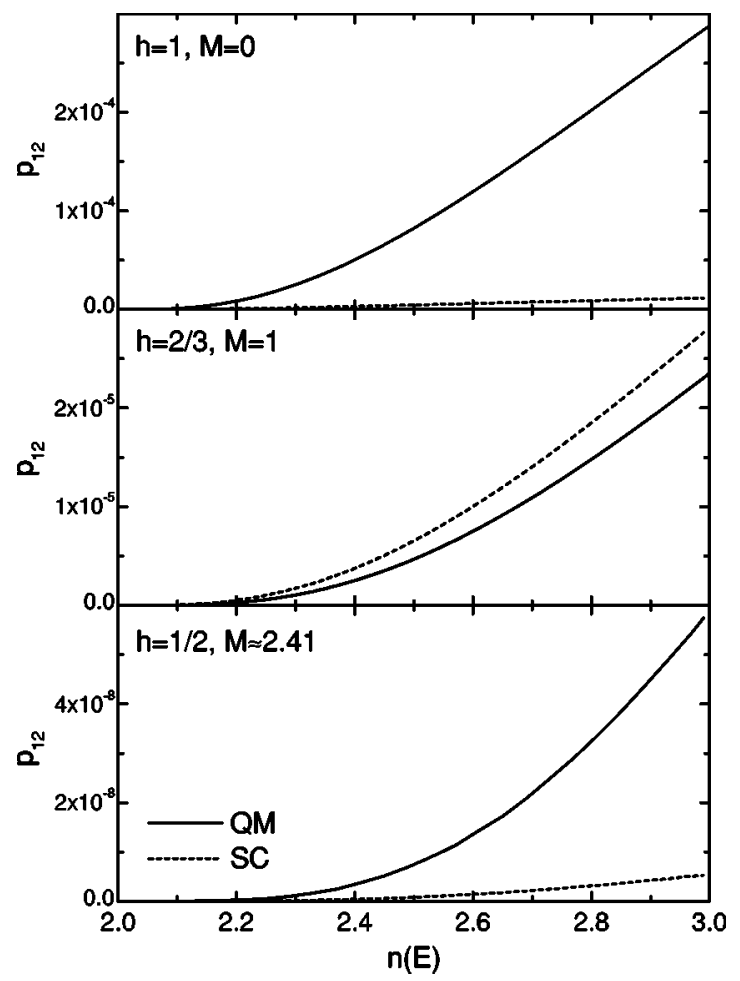

FIG. 14. Same as in Fig. 11, but in case B. There are no resonances in this case.

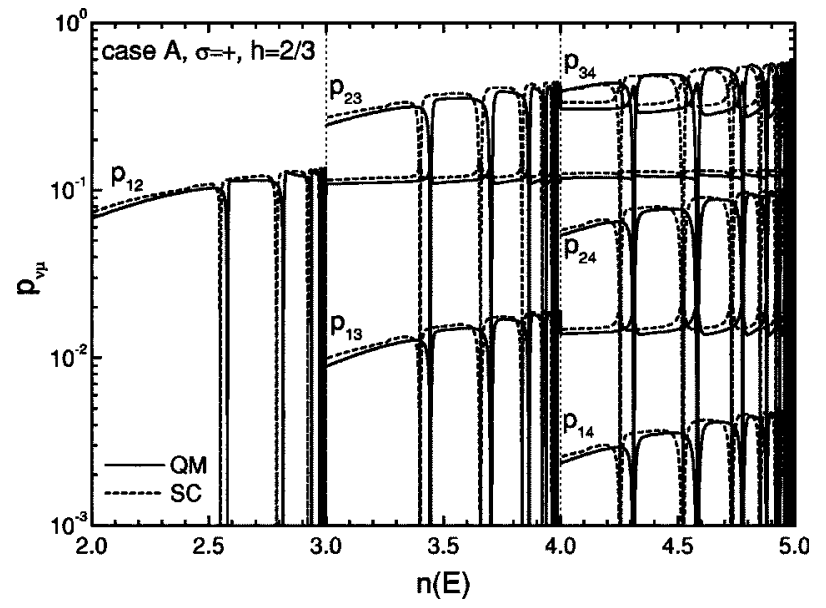

FIG. 15. The probabilities of various transitions for $h=2 / 3$ in case A, $\sigma=+$. All inelastic transitions in this energy range are shown. Vertical dotted lines indicate threshold energies $E=E_{\nu}$.

\section{CONCLUSIONS}

The main goal of this work was to investigate how the SC and QM results converge for the given class of systems when the asymptotic parameter $h$ tends to zero. The main conclusion is that this convergence is strongly affected by Stueckelberg's oscillations, i.e., by the interference effects. It is shown that the overall agreement between the SC and QM results for a wide spectrum of systems and processes in a wide energy range is surprisingly good even for $h \sim 1$. However, because of the oscillations the convergence is not monotonic, and in each particular case, i.e., for a given system and process, the SC results may be grossly in error even for small values of $h$ in some unfavorable situations where a destructive interference occurs. The main source of the discrepancy is a small $O(h)$ error in the interference phases, which is intrinsic to the leading order approximation. We stress that this error cannot be eliminated by simply including the so-called dynamic phase [2,3] into Stueckelberg's connection matrix (65), let alone higher order corrections that follow from the solution of the two-state linear model

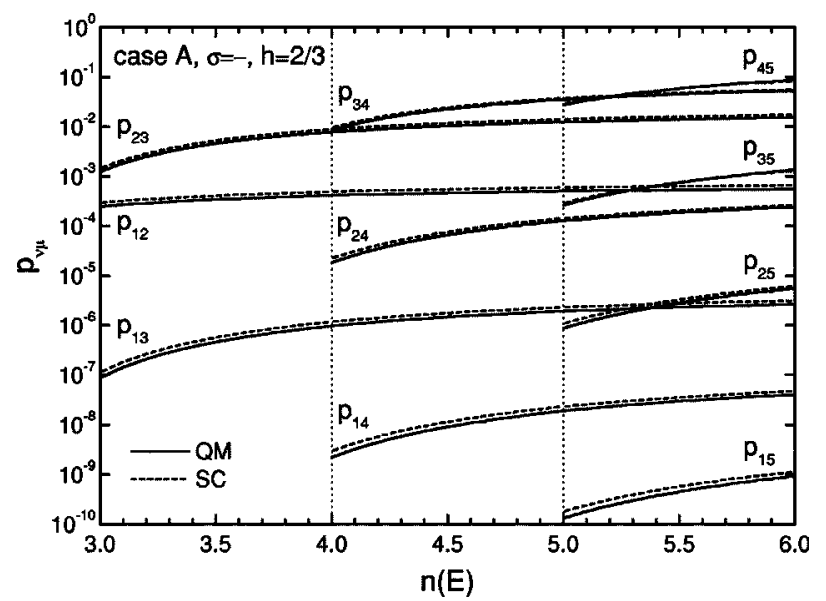

FIG. 16. Same as in Fig. 15, but in case A, $\sigma=-$. Resonances are too narrow, not resolved. 


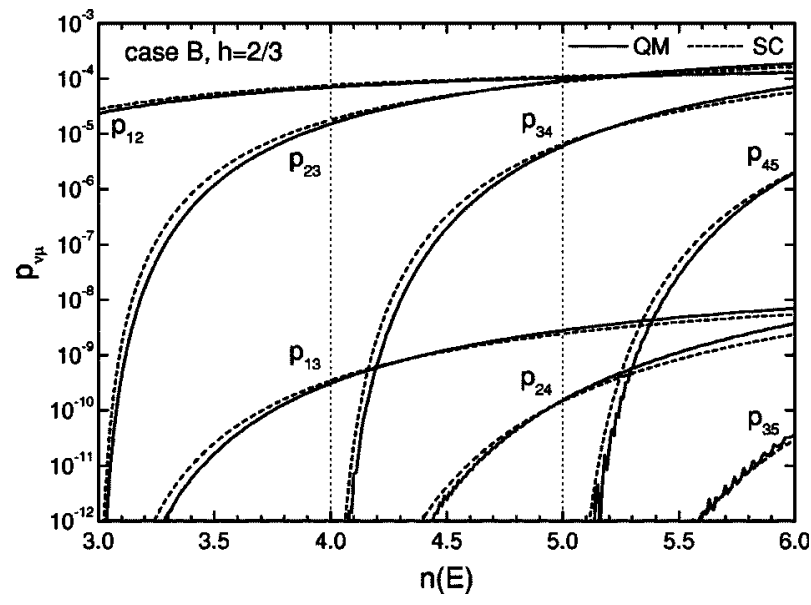

FIG. 17. Same as in Fig. 15, but in case B. Only transitions whose probabilities are larger than $10^{-12}$ are shown. The zigzag structures in the QM results indicate the lack of convergence caused by the roundoff errors. There are no resonances in this case.

[5], because there are other terms of the same order represented by the first order correction (42) to the primitive SC solutions (44). Of course, this conclusion is not a surprise. We have discussed oscillations in $h$, but similar oscillations exist in the dependence of transition probabilities on the impact parameter in time-dependent approaches, or on the total angular momentum in time-independent approaches. These oscillations are averaged out if one is interested only in total cross sections, but it is well-known that they strongly affect more detailed characteristics, such as partial cross sections. The interference effects similar to those discussed in this work have been detected earlier in three-body Coulomb systems in three dimensions [15]. It was shown that the widths of resonances oscillate as functions of the mass ratio $M$, and without these oscillations it is impossible to understand, e.g., the isotope dependence of the resonance widths in muonic molecules $p p \mu, d d \mu$, and $t t \mu$. This example suggests that our conclusion should remain valid also for the threedimensional case.

Our second result consists in the analysis of hidden crossings in the collinear three-body Coulomb problem and, in particular, in finding the complex $T$ series. Even though the mechanism of the complex $T$ series is formally similar to that of the usual $T$ series [6], namely, in both cases the nonadiabatic transitions occur near the extremum of the potential energy defining the motion in a "quantal" ("fast") variable, in the complex case there is no potential barrier on the real axis, which leads to a qualitatively different distribution of hidden crossings in the complex plane of the "classical" ("slow") variable, and hence to a different behavior of the transition probabilities. Whether the effects of the complex $T$ series can be found in the dynamics of physical systems in three dimensions remains an open question.

Finally, let us mention some directions in which we hope to continue these efforts. This work is intended to be the first part of a series. In the subsequent parts we plan to consider resonances [55] and fragmentation processes. This will complete the quantum mechanical and semiclassical study of the collinear three-body Coulomb problem. A similar study in the three-dimensional case should start with the analysis of hidden crossings. The first step in that direction has been made recently [56].

\section{ACKNOWLEDGMENTS}

O.I.T. thanks E. A. Solov'ev for introducing him to the phenomenon of hidden crossings and numerous discussions on the subject of this paper. This work was supported in part by a Grant-in-Aid for Scientific Research (C) from the Ministry of Education, Culture, Sports, Science and Technology, Japan, and also in part by the 21st Century COE program on "Coherent Optical Science."
[1] M. Born and R. Oppenheimer, Ann. Phys. (Leipzig) 84, 457 (1927).

[2] E. E. Nikitin and S. Ya. Umanskii, Theory of Slow Atomic Collisions (Springer-Verlag, Berlin, 1984).

[3] M. S. Child, Semiclassical Mechanics with Molecular Applications (Clarendon, Oxford, 1991).

[4] E. S. Medvedev and V. I. Osherov, Radiationless Transitions in Polyatomic Molecules (Springer-Verlag, Berlin, 1995).

[5] H. Nakamura, Nonadiabatic Transition: Concepts, Basic Theories, and Applications (World Scientific, Singapore, 2002).

[6] E. A. Solov'ev, Usp. Fiz. Nauk 157, 437 (1989) [Sov. Phys. Usp. 32, 228 (1989)].

[7] M. Born and V. Fock, Z. Phys. 51, 165 (1928).

[8] S. F. C. O'Rourke, B. S. Nesbitt, and D. S. F. Crothers, Adv. Chem. Phys. 103, 217 (1998).

[9] V. I. Osherov, in Nonadiabatic Transitions in Quantum Systems, edited by V. I. Osherov and L. I. Ponomarev (IPCP RAS, Chernogolovka, 2004), p. 124.

[10] Yu. N. Demkov and V. N. Ostrovsky, J. Phys. B 28, 403
(1995); V. N. Ostrovsky and H. Nakamura, J. Phys. A 30, 6939 (1997).

[11] O. I. Tolstikhin, S. Watanabe, and M. Matsuzawa, J. Phys. B 29, L389 (1996).

[12] D. Kato and S. Watanabe, Phys. Rev. A 56, 3687 (1997).

[13] M. Zamkov, E. P. Benis, C. D. Lin, T. G. Lee, T. Morishita, P. Richard, and T. J. M. Zouros, Phys. Rev. A 67, 050703 (2003).

[14] C.-N. Liu, A.-T. Le, T. Morishita, B. D. Esry, and C. D. Lin, Phys. Rev. A 67, 052705 (2003).

[15] O. I. Tolstikhin, I. Yu. Tolstikhina, and C. Namba, Phys. Rev. A 60, 4673 (1999).

[16] O. I. Tolstikhin and C. Namba, Phys. Rev. A 60, 5111 (1999).

[17] O. I. Tolstikhin and H. Nakamura, J. Chem. Phys. 108, 8899 (1998).

[18] F. D. Colavecchia, F. Mrugała, G. A. Parker and R. T. Pack, J. Chem. Phys. 118, 10387 (2003).

[19] O. I. Tolstikhin and C. Namba, CTBC-A Program to Solve the Collinear Three-Body Coulomb Problem: Bound States and Scattering below the Three-Body Disintegration Threshold, 
Research Report NIFS-779 (National Institute for Fusion Science, Toki, Japan, 2003). Available from bunken@ @ifs.ac.jp by request.

[20] L. Landau, Phys. Z. Sowjetunion 1, 88 (1932); 2, 46 (1932).

[21] E. C. G. Stueckelberg, Helv. Phys. Acta 5, 369 (1932).

[22] E. A. Solov'ev, Zh. Eksp. Teor. Fiz. 81, 1681 (1981) [Sov. Phys. JETP 54, 893 (1981)].

[23] E. A. Solov'ev, in The Physics of Electronic and Atomic Collisions, edited by L. J. Dubé et al., AIP Conf. Proc. No. 360 (AIP, Woodbury, NY, 1996), p. 471; also in Ref. [9], p. 150.

[24] F. T. Smith, J. Chem. Phys. 31, 1352 (1959).

[25] K. L. Baluja, P. G. Burke, and L. A. Morgan, Comput. Phys. Commun. 27, 299 (1982).

[26] P. G. Burke and H. M. Schey, Phys. Rev. 126, 147 (1962).

[27] M. Gailitis, J. Phys. B 9, 843 (1976).

[28] C. J. Noble and R. K. Nesbet, Comput. Phys. Commun. 33, 399 (1984)

[29] F. T. Smith, Phys. Rev. 120, 1058 (1960).

[30] J. Macek, J. Phys. B 1, 831 (1968).

[31] C. D. Lin, Adv. At. Mol. Phys. 22, 77 (1986); Phys. Rep. 257, 1 (1995).

[32] Advances in Molecular Vibrations and Collision Dynamics, edited by J. M. Bowman (JAI Press Inc., London, 1994), Vols. $2 \mathrm{~A}$ and $2 \mathrm{~B}$.

[33] R. E. Langer, Phys. Rev. 51, 669 (1937).

[34] Yu. N. Demkov, in The Physics of Electronic and Atomic Collisions, ICPEAC-V. Invited Papers, edited by L. M. Branscomb (Nauka, Leningrad, 1967), p. 186.

[35] G. Herzberg and H. C. Longuet-Higgins, Discuss. Faraday Soc. 35, 77 (1963).

[36] O. I. Tolstikhin, V. N. Ostrovsky, and H. Nakamura, Phys. Rev. A 58, 2077 (1998).

[37] A. Zwaan, Arch. Neerland. Ser. III A 12, 1 (1929).
[38] J. Heading, An Introduction to Phase-Integral Methods (Methuen, London, 1962).

[39] D. S. F. Crothers and P. G. Mulligan, in Ref. [9], p. 93.

[40] D. S. F. Crothers, Adv. Phys. 20, 405 (1971).

[41] F. W. J. Olver, Asymptotics and Special Functions (Academic Press, New York, 1974).

[42] M. V. Fedoryuk, Asymptotic Analysis: Linear Ordinary Differential Equations (Springer-Verlag, Berlin, 1993).

[43] S. Yu. Ovchinnikov and E. A. Solov'ev, Zh. Eksp. Teor. Fiz. 90, 921 (1986) [Sov. Phys. JETP 63, 538 (1986)].

[44] E. A. Solov'ev, Zh. Eksp. Teor. Fiz. 90, 1165 (1986) [Sov. Phys. JETP 63, 678 (1986)].

[45] S. Yu. Ovchinnikov and E. A. Solov'ev, Zh. Eksp. Teor. Fiz. 91, 477 (1986) [Sov. Phys. JETP 64, 280 (1986)].

[46] T. P. Grozdanov and E. A. Solov'ev, Phys. Rev. A 51, 2630 (1995).

[47] V. I. Savichev and E. A. Solov'ev, Phys. Lett. A 205, 184 (1995).

[48] D. Jakimovski, V. I. Savichev, and E. A. Solov'ev, Phys. Rev. A 54, 2962 (1996).

[49] R. K. Janev, Phys. Rev. A 55, R1573 (1997); 55, 4285 (1997).

[50] M. P. Strand and W. P. Reinhardt, J. Chem. Phys. 70, 3812 (1979).

[51] D. I. Abramov, S. Yu. Ovchinnikov, and E. A. Solov'ev, Phys. Rev. A 42, 6366 (1990).

[52] J. H. Macek and S. Yu. Ovchinnikov, Phys. Rev. A 50, 468 (1994).

[53] J. S. Briggs, V. I. Savichev, and E. A. Solov'ev, J. Phys. B 33, 3363 (2000).

[54] C. M. Bender and T. T. Wu, Phys. Rev. 184, 1231 (1969).

[55] G. V. Sitnikov and O. I. Tolstikhin (unpublished).

[56] E. A. Solov'ev and O. I. Tolstikhin, J. Phys. B 34, L439 (2001). 